\title{
( ) Angebots-, Nutzungs- \\ und Bezugsstrukturen \\ elektronischer \\ Fachinformation in \\ Deutschland
}

Aus: ABI-Technik,

Band 23, Heft 2, Seiten 122-142,

ISSN (Online) 2191-4664,

ISSN (Print) 0720-6763,

DOI: https://doi.org/10.1515/

ABITECH.2003.23.2.122

\author{
Ein Beitrag von Andreas Degkwitz und Heike Andermann
}

Mit dem Übergang vom analogen zum digitalen Medium haben sich die Bezugsstrukturen und das Angebot an elektronischer Fachinformation der Bibliotheken nachhaltig verändert. In den vorliegenden Untersuchungen wird das Angebot elektronischer Zeitschriften und Datenbanken und die Nutzung elektronischer Zeitschriften in fünf ausgewählten Fachgebieten und in verschiedenen Bibliothekstypen dargelegt. Darüber hinaus werden die derzeitigen Bezugsstrukturen beschrieben, sowie die Ergebnisse einer Befragung der Konsortien zu Zielsetzungen, Vertragsformen und Geschäftsmodellen dargestellt. Chancen und Risiken der konsortialen Bezugsform werden erörtert.

With the transition from the printed to the digital medium the structures of purchasing digital information and the offer of scientific information in the libraries changed strongly. In the available examination the offer of electronic journals and databases and the usage of electronic journals in five selected disciplines and in different types of libraries is evaluated. Further more the current purchasing structures and the results of interviews with consortia in regard to objectives, forms of contracts and pricing models are described. Chances and risks of consortia purchasing are discussed.

\section{Einleitung}

Zielsetzung des von der Deutschen Forschungsgemeinschaft geförderten Projektes "Perspektiven für den Bezug elektronischer Fachinformation in der Bundesrepublik Deutschland" ist eine Bestandsaufnahme und Bewertung gegenwärtiger Bezugsformen elektronischer Informationsressourcen in Deutschland, sowie eine Beschreibung neuer Modelle künftiger Bezugs- und Bereitstellungsstrukturen elektronischer Fachinformation. Neben einer allgemeinen Einführung in die gegenwärtige Problematik der wissenschaftlichen Informationsversorgung werden die Ergebnisse der in dem Projekt durchgeführten Untersuchungen zu Angebots- und Nutzungsstrukturen elektronischer Fachinformation vor- gestellt. Darüber hìnaus werden die derzeitigen Bezugsstrukturen in Deutschland beschrieben und die Ergebnisse einer Befragung der Konsortien dargelegt.

\section{Problemaufriss}

Wissenschaftliche Bibliotheken übernehmen eine zentrale Funktion in der Informationsversorgung, indem sie ein umfangreiches und möglichst vollständiges Angebot an wissenschaftlicher Fachinformation für Lehre und Forschung bereitstellen. Zwischen dem Anspruch an vollständiger Informationsversorgung und den tatsächlichen finanziellen Möglichkeiten zur Beschaffung dieser Information ist seit einigen Jahren eine zunehmende Diskrepanz zu beobachten. Bibliotheken sind immer weniger in der Lage, die wissenschaftliche Informationsversorgung im traditionellen Verständnis aufrechtzuerhalten. Hierfür sind mehrere Faktoren von Bedeutung, die hier nur kurz benannt werden können. Die Entwicklung der Wissenschaften ist durch schnelles Wachstum an wissenschaftlicher Information, durch zunehmende Spezialisierung und durch eine rasche Alterung vor allem des naturwissenschaftlich-technischen Wissens gekennzeichnet. Hinzu kommt der wachsende Publikationsdruck der NachwuchswissenschaftlerInnen, die für ihr berufliches Fortkommen möglichst zahlreiche Artikel in Fachzeitschriften publizieren müssen. Diese Entwicklung hat die Herausgabe immer neuer Zeitschriften befördert [1], die durch die Bibliotheken erworben bzw. lizenziert werden. Dem gestiegenen Angebot an wissen- 
schaftlicher Information und den noch steigenden Kosten für diese, steht auf der Seite der Bibliotheken ein rückläufiger Etat gegenüber mit der Folge, dass Bibliotheken gezwungen werden, Zeitschriftenexemplare in großem Umfang abzubestellen. Die gegenwärtige Situation der Bibliotheken wird auch mit dem Begriff der Krise der wissenschaftlichen Informationsversorgung belegt. Gemeint ist, dass die hohen und noch steigenden Kosten für die Versorgung mit gedruckter und elektronischer Fachinformation (hier elektronische Zeitschriften) insbesondere in den naturwissenschaftlich-technischen Fächern zu einer Gefährdung des Bestandsaufbaus und damit der Informationsversorgung in den anderen Fachgebieten führt.

\section{Die Bedeutung der Zeitschriften in der wissen- schaftlichen Infor- mationsversorgung}

Der Markt für wissenschaftliche Information in den naturwissenschaftlich-technischen Fachgebieten zeichnet sich durch ein verhältnismäßig geringes Angebot an qualitativ hochwertigen und ein umfangreiches Angebot an mittelmäßigen Forschungsergebnissen aus [2]. Die Verlage wiederum verfügen über eine Vielzahl von Zeitschriften unterschiedlichen Renommees, in denen ein Artikel platziert werden kann. Das Renommee einer Zeitschrift ist von entscheidender $\mathrm{Be}$ deutung, handelt es sich hierbei doch um einen wichtigen Indikator der Zeitschriftenqualität. Reputation, Reichweite und Ansehen der Zeitschrift entscheiden über die Akzeptanz bei den WissenschaftlerInnen, d. h. als Kommunikationsplattform übernehmen Zeitschriften für die wissenschaftliche Gemeinschaft eine wichtige Netzwerkfunktion. In diesem Sinne ist es für die WissenschaftlerInnen von großer $\mathrm{Be}$ - deutung, wissenschaftliche Neuheiten in einer Zeitschrift mit hohem Renommee zu publizieren. Dabei erfüllt die Zeitschrift unterschiedliche Funktionen:

- Verbreitung wissenschaftlicher Erkenntnisse

- Entdeckungspriorität:

Durch die Veröffentlichung eines Aufsatzes in einer anerkannten Zeitschrift erheben die WissenschaftlerInnen Anspruch auf die wissenschaftliche Entdeckung und die Urheberschaft auf die gewonnenen Erkenntnisse.

- Qualitätskontrolle: Durch das Peer-Review-Verfahren wird eine Auswahl unter den zu veröffentlichenden Artikeln getroffen. Die Zeitschrift stellt somit einen Qualitätsfilter dar, der den WissenschaftlerInnen vor dem Hintergrund einer zunehmenden Publikationsflut eine Qualitätsorientierung ermöglicht.

- Qualitatives Gütesiegel: WissenschaftlerInnen sind schon aus zeitlichen Gründen auf den Qualitätsfilter, den die Fachzeitschrift darstellt, angewiesen. Die hierarchisierende Einordnung der Artikel in den Zeitschriften ermöglicht darüber hinaus eine Einschätzung des Status der WissenschaftlerInnen innerhalb der wissenschaftlichen Gemeinschaft.

\section{Vertriebsstrate- gien/Ziele der Anbieterseite}

Mit der Entwicklung der elektronischen Zeitschriften haben auch die Verlage ihre Vertriebsstrategien geändert, um den Absatz ihrer Produkte zu sichern bzw. ihren Gewinn zu steigern. Kennzeichnend für die gegenwärtige Bezugssituation sind folgende Faktoren:

- Koppelung des elektronischen Exemplars an das Printexemplar einer Zeitschrift

- Bündelung der Zeitschriften zu sogenannten Zeitschriftenpaketen

○ Vertraglich festgelegte Abbestellquoten für Printexemplare von Zeitschriften
Bibliotheken beziehen elektronische Zeitschriften gegenwärtig überwiegend im Parallelbezug in der Print- und in der elektronischen Ausgabe für einen jeweils zu definierenden Abnehmerkreis. Auf der Anbieterseite werden die Produkte nicht einzeln verkauft, sondern in so genannten Titelpaketen zusammengefasst und vertrieben. Dadurch gelingt es den Verlagen, neben ihrem oftmals kleinen Spektrum an Zeitschriften mit hohem Renommee, ein erheblich größeres Spektrum an Zeitschriften, in denen qualitativ geringerwertige Artikel publiziert werden, an die institutionellen Abnehmer zu vertreiben. Mit Hilfe der Titelpakete werden auf diese Weise auch qualitativ schlechtere Zeitschriften bei der Zielgruppe eingeführt. Darüber hinaus bestehen die Verlage auf der Festlegung von sogenannten Abbestellquoten für Printexemplare von Zeitschriften: Bibliotheken verpflichten sich, eine festgelegte Höhe von Zeitschriftenabbestellungen (die in der Regel sehr niedrig ist) nicht zu überschreiten.

Die Preisbildung für diese Produkte erfolgt auf der Basis der Printabonnements (Basiskosten) plus Kosten für die elektronische Parallelversion (Lizenzkosten). Derzeitig ist der Bezug ausschließlich elektronischer Publikationen durch die Bibliotheken nicht durchgängig verbreitet, nimmt aber stetig zu.

Verlage haben ein starkes Interesse, die traditionelle Wertschöpfungskette im Informationsmarkt auch im elektronischen Umfeld aufrechtzuerhalten und ihre Position zu stabilisieren. Seit mehreren Jahren ist bei einigen Verlagen ein verändertes Marktverhalten festzustellen. $\mathrm{Zu}$ beobachten ist eine zunehmend aggressive Expansionsstrategie, die sich darin äußert, dass Verlage mit einem gleichartigen Fächerspektrum von größeren Verlagen aufgekauft [3] werden. Die Folge ist eine 
- Angebots-, Nutzungs- und Bezugsstrukturen elektronischer Fachinformation in Deutschland zunehmende Konzentration auf einige Anbieter im Informationsmarkt (Oligopol). Mit dieser Strategie werden mehrere Ziele verfolgt: der konkurrierende Verlag kann vom Markt gedrängt werden und das Verlagsspektrum an wissenschaftlichen Zeitschriften wird integriert. Durch die Übernahme von Verlagen lassen sich Preiserhöhungen für die wissenschaftlichen Zeitschriften leichter durchsetzen, da der Konkurrent auf dem Markt nicht länger agiert. Seinen Ausdruck findet dies in den hohen und noch steigenden Kosten für den Bezug von Printzeitschriften und elektronischen Zeitschriften.

Gegenwärtig ist die Situation dadurch gekennzeichnet, dass öffentlich finanzierte Hochschulen die mit öffentlichen Mitteln finanzierte wissenschaftliche Information von den Verlagen zurück erwerben müssen [4]. Dies geschieht in der Regel durch die Konsortien. Die konsortiale Bezugsform dürfte sich jedoch nur als eine kurzfristig erfolgreiche Strategie erweisen mit dem Ziel, ein Mehr an Information durch indirekte Rabattierungsmöglichkeiten zu erhalten. In einer mittelbis langfristigen Perspektive erscheint eine Neuordnung der Wertschöpfungskette im digitalen Umfeld jedoch unerlässlich, um die Informationsversorgung dauerhaf sicherzustellen.

In den folgenden Kapiteln werden die im Rahmen des Projektes durchgeführten Untersuchungsergebnisse dargestellt. Mit Hilfe einer Angebotsanalyse wurde der Umfang und der Inhalt an elektronischer Fachinformation in einer repräsentativen geschichteten Stichprobe von wissenschaftlichen Bibliotheken unterschiedlichen Typs in verschiedenen Fachgebieten untersucht. Darüber hinaus wurde die Nutzung elektronischer Zeitschriften in verschiedenen Fachgebieten untersucht. Die Konsortialstrukturen und die Zielsetzungen verschiedener Kon- sortien wurden mit Hilfe eines standardisierten Fragebogens ermittelt.

\section{Untersuchungen im Rahmen des Projektes}

\subsection{Struktur der Verträge}

Grundsätzlich ist zwischen dem Einzelbezug und dem Konsortialbezug zu unterscheiden. Bei dem Einzelbezug lassen sich die Kernelemente Berechnungsgrundlage, Archivzugriff und $\mathrm{Zu}$ griffsbeschränkung unterscheiden.

Ihnen können folgende Merkmale zugeordnet werden:

Berechnungsgrundlage

- Lizenzgebühren für elektronischen Zugriff in PrintAbonnement enthalten

- Lizenzgebühren für elektronischen Zugriff zusätzlich zu Print-Abonnement

- Bezug über Einzelabrechnung pro Artikel (pay-perview)

- Alternativer Bezug (PrintAusgabe oder elektronischer Bezug)

Archivzugriff:

- Archivzugriff in Lizenz für elektronische Nutzung enthalten

- Archivzugriff nur mit elektronischer Lizenz

Zugriffsbeschränkung:

- Geographische Beschränkung

○ IP-gesteuerte Zugriffsregelung

○ Beschränkung simultaner Zugriffe

Bei dem Konsortialbezug lassen sich hinsichtlich der Berechnungsgrundlage und des Leistungsumfangs folgende Vertragsmodalitäten unterscheiden

Berechnungsgrundlage

- Kalkulationsbasis PrintAbonnement (Primärprodukt gedruckte Zeitschriften)
- Lizenzpreis nach Anzahl der Konsorten

- Lizenzpreis nach potenziellen Nutzern

Leistungsumfang:

- cross access: Die in einem Konsortium zusammengeschlossenen Bibliotheken haben Zugriff auf die elektronischen Versionen der Zeitschriften, für die im Konsortium mindestens ein Print-Exemplar vorhanden ist.

- additional access: Bibliotheken eines Konsortiums haben Zugriff auf das gesamte elektronische Spektrum wissenschaftlicher Zeitschriften eines Verlags.

- dauerhafter Zugriff: Bibliotheken eines Konsortiums haben auch nach Ablauf der Lizenzvertrages Zugriff auf den im Lizenzvertrag festgelegten Vertragsumfang.

Die Optionen cross access und additional access werden von den Bibliotheken als indirekte Rabattierungsmöglichkeit angesehen, da hierüber ein Zugriff auf eine gröBere Menge an elektronischer Fachinformation möglich ist und die Kosten/Einheit gesenkt werden können.

\subsection{Marktdurchdringung} und Produktrelevanz nach Bibliothekstyp

In einer repräsentativen geschichteten Stichprobe wurde der Bestand an elektronischer Fachinformation in 54 Bibliotheken unterschiedlichen Typs in fünf exemplarisch ausgewählten Fachgebieten untersucht. Bei den Bibliotheken handelt es sich um Universitätsbibliotheken, Fachhochschulbibliotheken Nationalbibliotheken und $\mathrm{Re}$ gionalbibliotheken [5]. Universitätsbibliotheken sind die Hauptabnehmer elektronischer Zeitschriften. Von den in der Stichprobe enthaltenen 3.679 Zeitschriftentiteln entfallen auf die einzelnen Bibliothekstypen:

\begin{tabular}{|c|c|c|c|}
\hline Universitätsbibliothek & $\begin{array}{c}\text { Fachhochschui- } \\
\text { bibliothek }\end{array}$ & Nationalbibliothek & Regionalbıbliothek \\
\hline 3.585 & 1.946 & 2.394 & 1.571 \\
\hline $97,40 \%$ & $53 \%$ & $65 \%$ & $43 \%$ \\
\hline
\end{tabular}

Tab. 1: Titel/Bibliothekstyp 
Die Universitätsbibliotheken halten mit $97 \%$ das größte Spektrum an elektronischen Zeitschriften vor, gefolgt von den Nationalbibliotheken mit $65 \%$. Hiervon entfallen auf die einzelnen Fachgebiete:

\begin{tabular}{|l|c|c|c|c|c|}
\hline \multicolumn{1}{|c|}{ Fachgebiet } & $\begin{array}{c}\text { Chemie/ } \\
\text { Pharmazie }\end{array}$ & Elektrotechnik & Geschichte & Mathematik & Psychologie \\
\hline kostenpflichtig & $86 \%$ & $83 \%$ & $57 \%$ & $72 \%$ & $43 \%$ \\
\hline kostenlos & $13 \%$ & $14 \%$ & $28 \%$ & $22 \%$ & $8 \%$ \\
\hline keine Angabe & $1 \%$ & $3 \%$ & $15 \%$ & $6 \%$ & $50 \%$ \\
\hline
\end{tabular}

Bei den Anbietern handelt es sich um kommerzielle Verlage, Fachgesellschaften und Universitätsverlage. $48 \%$ der Zeitschriftenexemplare werden von großen kommerziellen Verlagen bezogen. Auf die exemplarisch ausgewählten kommerziellen Verlage entfallen folgende Anteile:

\begin{tabular}{|c|c|c|c|c|c|c|}
\hline Verlage & Elsevier & Springer & Kluwer & Wiley & $\begin{array}{c}\text { Academic } \\
\text { Press }\end{array}$ & Summe \\
\hline $\begin{array}{c}\text { Anzahl der } \\
\text { Exemplare }\end{array}$ & 7.940 & 2.311 & 5.729 & 3.396 & 6.043 & 25.419 \\
\hline $\begin{array}{c}\text { Anteil am } \\
\text { Gesamtumfang der } \\
\text { Exemplare in } \\
\text { (n=53.147) }\end{array}$ & $15 \%$ & $4 \%$ & $11 \%$ & $6 \%$ & $11 \%$ & $48 \%$ \\
\hline
\end{tabular}

Auf die einzelnen wissenschaftlichen Bibliothekstypen entfallen folgende Anteile an Zeitschriftentiteln:

Bibliothekstyp: Universitätsbibliothek

\begin{tabular}{|c|c|c|c|c|c|}
\hline Elsevier & Springer & Kluwer & Wiley & $\begin{array}{c}\text { Academic } \\
\text { Press }\end{array}$ & Summe \\
\hline 5.422 & 4.126 & 4.035 & 2.904 & 1.353 & 17.840 \\
\hline
\end{tabular}

Bibliothekstyp: Fachhochschulbibliothek

\begin{tabular}{|c|c|c|c|c|c|}
\hline Elsevier & Springer & Kluwer & Wiley & $\begin{array}{c}\text { Academic } \\
\text { Press }\end{array}$ & Summe \\
\hline 1.392 & 1.339 & 888 & 244 & 541 & 4.404 \\
\hline
\end{tabular}

Bibliothekstyp: Nationalbibliothek

\begin{tabular}{|c|c|c|c|c|c|}
\hline Elsevier & Springer & Kłuwer & Wiley & Academic Press & Summe \\
\hline 464 & 110 & 260 & 246 & 139 & 1.219 \\
\hline
\end{tabular}

Bibliothekstyp: Regionalbibliothek

\begin{tabular}{|c|c|c|c|c|c|}
\hline Elsevier & Springer & Kluwer & Wiley & $\begin{array}{c}\text { Academic } \\
\text { Press }\end{array}$ & Summe \\
\hline 662 & 468 & 546 & 2 & 278 & 1.956 \\
\hline
\end{tabular}

Universitätsbibliotheken halten das größte Spektrum an Zeitschriftenexemplaren vor. Ihr Anteil an den exemplarisch ausgewählten kommerziellen Verlagen liegt bei $70 \%$.

\subsection{Impact Faktor/Biblio- theks typ/Fachgebiet}

502 der insgesamt 3.679 Zeitschriftentitel werden im Web of Science des Institute of Scientific Information (ISI) nachgewiesen. Für diese Zeitschriften ist die Ermittlung des Impact Faktors möglich. 15 Zeitschriften mit einem Impact Faktor von über zehn sind den Fachgebieten der Chemie/Pharmazie und Psychologie zuzuordnen und werden überwiegend vom Bibliothekstyp Universitätsbibliothek bereitgestellt.

\begin{tabular}{|c|c|c|c|c|c|}
\hline \multirow[t]{2}{*}{ Zeitschriftentitel } & \multirow[t]{2}{*}{$\begin{array}{l}\text { Impact } \\
\text { Faktor }\end{array}$} & \multicolumn{4}{|c|}{$\begin{array}{l}\text { Anzahl der } \\
\text { Bibliotheken/Bibliothekstyp }\end{array}$} \\
\hline & & UB & FHB & NB & RB \\
\hline Annual Review of Biochemistry & 43,42 & 3 & 0 & 0 & 0 \\
\hline Annual Review of Neuroscience & 26,67 & 4 & 0 & 0 & 0 \\
\hline Nature & 25,81 & 20 & 0 & 0 & 0 \\
\hline Pharmacological Review & 25,35 & 6 & 0 & 2 & 0 \\
\hline Science & 23,87 & 0 & 0 & 1 & 0 \\
\hline Chemical Review & 20,03 & 17 & 3 & 1 & 2 \\
\hline $\begin{array}{c}\text { Annual Review for Pharmacology and } \\
\text { Toxicology }\end{array}$ & 19,28 & 3 & 0 & 0 & 0 \\
\hline Surface Science Reports & 14,95 & 11 & 3 & 1 & 2 \\
\hline Behavioral and Brain Sciences & 14,25 & 12 & 0 & 1 & 0 \\
\hline Accounts of Chemical Research & 13,26 & 17 & 3 & 1 & 2 \\
\hline Trends in Biochemical Sciences & 13,24 & 9 & 3 & 1 & 2 \\
\hline Archive of General Psychiatry & 11,78 & 4 & 0 & 0 & 0 \\
\hline $\begin{array}{c}\text { Proceedings of the National Academy } \\
\text { of Sciences }\end{array}$ & 10,78 & 17 & 0 & 1 & 0 \\
\hline Chemical Society Reviews & 10,74 & 14 & 1 & 1 & 0 \\
\hline Trends in Pharmacological Sciences & 10,37 & 9 & 3 & 1 & 2 \\
\hline $\begin{array}{l}\text { Summe der Bibliotheken } \\
\text { /Bibliothekstyp }\end{array}$ & & 146 & 16 & 11 & 10 \\
\hline $\begin{array}{l}\text { Summe der Bibliotheken } \\
\text { /Bibliothekstyp in \% }\end{array}$ & & $80 \%$ & $9 \%$ & $6 \%$ & $5 \%$ \\
\hline
\end{tabular}

22 der insgesamt 502 Zeitschriften mit einem Impact Faktor von über 5 entstammen überwiegend den Fachgebieten Chemie/Pharmazie und Psychologie. Auch diese Zeitschriftentitel werden überwiegend vom Bibliothekstyp Universitätsbibliothek bereitgestellt (Tab. 9)

Die Lizenzierung von Zeitschriften mit einem hohen Impact Faktor erfolgt zu $80 \%$ durch Universitätsbibliotheken. Bei den Anbietern der oben aufgeführten Zeitschriften handelt es um amerikanische Fachgesellschaften, die HighWirePress als Service Provider für ihre elektronischen Publikationen nutzen und kommerzielle Verlage wie Elsevier Science, Wiley VCH, Blackwell Publisher und SpringerLINK.
Angebots-, Nutzungs- und Bezugsstrukturen elektronischer Fachinformation in Deutschland

\section{Tab. 8: Zeitschriftentitel/} Impact Faktor/Bibliothekstyp $>10$ 


\begin{tabular}{|c|c|c|c|c|c|}
\hline \multirow[t]{2}{*}{ Zeitschriftentitel } & \multirow[t]{2}{*}{$\begin{array}{l}\text { Impact } \\
\text { Faktor }\end{array}$} & \multicolumn{4}{|c|}{$\begin{array}{l}\text { Anzahl der } \\
\text { Bibliotheken/Bibliothekstyp }\end{array}$} \\
\hline & & UB & FHB & NB & RB \\
\hline Annual Review of Medicine & 9,81 & 4 & 0 & 0 & 0 \\
\hline Annual Review of Physical Chemistry & 9,23 & 2 & 0 & 0 & 0 \\
\hline Brain Research Reviews & 9,21 & 11 & 3 & 1 & 2 \\
\hline $\begin{array}{c}\text { Journal of Physical and Chemical } \\
\text { Reference Data }\end{array}$ & 8,75 & 8 & 1 & 1 & 0 \\
\hline $\begin{array}{l}\text { Angewandte Chemie Internationale } \\
\text { Edition }\end{array}$ & 8,54 & 1 & 0 & 0 & 0 \\
\hline Mass Spectrometry Reviews & 7,6 & 20 & 2 & 2 & 0 \\
\hline Journal of Biological Chemistry & 7,36 & 5 & 0 & 0 & 0 \\
\hline Current Opinion in Chemical Biology & 7,04 & 8 & 3 & 1 & 2 \\
\hline American Journal of Psychiatry & 6,57 & 7 & 0 & 1 & 0 \\
\hline Pharmacology \& Therapeutics & 6,48 & 7 & 2 & 1 & 0 \\
\hline Molecular Microbiology & 6,33 & 6 & 0 & 1 & 0 \\
\hline $\begin{array}{l}\text { Journal of the American Chemical } \\
\text { Society }\end{array}$ & 6,02 & 17 & 3 & 1 & 2 \\
\hline Aldrichimica Acta & 5,9 & 23 & 11 & 2 & 2 \\
\hline Annual Review of Psychology & 5,85 & 3 & 0 & 0 & 0 \\
\hline Chemistry \& Biology & 5,71 & 9 & 3 & 1 & 2 \\
\hline Molecular Pharmacology & 5,67 & 5 & 0 & 1 & 0 \\
\hline Advanced Materials & 5,52 & 15 & 1 & 1 & 0 \\
\hline $\begin{array}{c}\text { European Physical Journal C: } \\
\text { Particles and Fields }\end{array}$ & 5,4 & 19 & 5 & 0 & 1 \\
\hline Natural Product Reports & 5,29 & 6 & 0 & 1 & 0 \\
\hline Journal of Combinatorial Chemistry & 5,23 & 17 & 3 & 1 & 2 \\
\hline Journal of Cognitlve Neuroscience & 5,11 & 4 & 0 & 0 & 0 \\
\hline $\begin{array}{c}\text { Progress in Nuclear Magnetic } \\
\text { Reconance Spectroscopy }\end{array}$ & 5,06 & 32 & 6 & 2 & 4 \\
\hline $\begin{array}{c}\text { Summe der } \\
\text { Bibliotheken / Bibliothekstyp }\end{array}$ & & 229 & 38 & 16 & 15 \\
\hline $\begin{array}{c}\text { Summe der Bibliotheken/ } \\
\text { Bibliothekstyp in } \%\end{array}$ & & $77 \%$ & $13 \%$ & $5 \%$ & $5 \%$ \\
\hline
\end{tabular}

Tab. 9: Zeitschriftentitel/ Bibliothekstyp/Impact Faktor $<10$

\subsection{Datenbankprodukte}

Universitätsbibliotheken bieten das größte Spektrum an Datenbanken an. In der Stichprobe befinden sich 86 kostenpflichtige und 35 frei verfügbare fachbezogene Datenbankprodukte $(n=121)$, die sich wie folgt auf die Fachgebiete verteilen:

\begin{tabular}{|c|c|c|c|c|c|}
\hline Fachgebiet & $\begin{array}{c}\text { Chemie/ } \\
\text { Pharmazie }\end{array}$ & Psychologie & Elektrotechnik & Geschichte & Mathematik \\
\hline $\begin{array}{c}\text { Anzahl der } \\
\text { fachbezogenen } \\
\text { Datenbanken }\end{array}$ & 58 & 7 & 20 & 22 & 14 \\
\hline Angaben in \% & $48 \%$ & $6 \%$ & $16 \%$ & $19 \%$ & $11 \%$ \\
\hline
\end{tabular}

Tab. 10: Datenbanken/ Insgesamt beträgt die Anzahl Fachgebiet der von den Bibliotheken lizenzierten Datenbankprodukte in der Stichprobe 640. Von den Universitätsbibliotheken werden $81 \%$ dieser Datenbankprodukte bereitgestellt.

Auf die einzelnen Fachgebiete und auf die Bibliothekstypen entfallen folgende Anteile:

Fachgebiet Chemie/Pharmazie (Anzahl der kostenpflichtigen Datenbanken)

\begin{tabular}{|c|c|c|c|c|}
\hline Fachgebiet Chemie/Pharmazie & UB & NB & RB & FHB \\
\hline Anzahl der beziehenden Bibliotheken & 24 & 1 & 3 & 14 \\
\hline $\begin{array}{c}\text { Anzahl der fachbezogenen } \\
\text { Datenbanken }\end{array}$ & 203 & 4 & 6 & 26 \\
\hline
\end{tabular}

Tab. 11

Fachgebiet Psychologie [6] (Anzahl der kostenpflichtigen Datenbanken)

\begin{tabular}{|c|c|c|c|c|}
\hline Fachgebiet Psychologie & UB & NB & RB & FHB \\
\hline $\begin{array}{c}\text { Anzahl der beziehenden } \\
\text { Bibliotheken }\end{array}$ & 23 & $\cdots$ & 3 & 5 \\
\hline $\begin{array}{c}\text { Anzahl der fachbezogenen } \\
\text { Datenbanken }\end{array}$ & 52 & $\cdots$ & 2 & 1 \\
\hline
\end{tabular}

Tab. 12

Fachgebiet Mathematik

\begin{tabular}{|c|c|c|c|c|}
\hline Fachgebiet Mathematik & UB & NB & RB & FHB \\
\hline $\begin{array}{c}\text { Anzahl der beziehenden } \\
\text { Bibliotheken }\end{array}$ & 21 & $\cdots$ & 1 & 9 \\
\hline $\begin{array}{c}\text { Anzahl der fachbezogenen } \\
\text { Datenbanken }\end{array}$ & 69 & $\cdots$ & 5 & 11 \\
\hline
\end{tabular}

Tab. 13

Fachgebiet Geschichte

\begin{tabular}{|c|c|c|c|c|}
\hline Fachgebiet Geschichte & UB & NB & RB & FHB \\
\hline $\begin{array}{c}\text { Anzahl der beziehenden } \\
\text { Bibliotheken }\end{array}$ & 22 & 1 & 2 & 11 \\
\hline $\begin{array}{c}\text { Anzahl der fachbezogenen } \\
\text { Datenbanken }\end{array}$ & 93 & 11 & 3 & 14 \\
\hline
\end{tabular}

Tab. 14

Fachgebiet Elektrotechnik

\begin{tabular}{|c|c|c|c|c|}
\hline Fachgebiet Elektrotechnik & UB & NB & RB & FHB \\
\hline $\begin{array}{c}\text { Anzahl der beziehenden } \\
\text { Bibliotheken }\end{array}$ & 23 & 1 & 1 & 12 \\
\hline $\begin{array}{c}\text { Anzahl der fachbezogenen } \\
\text { Datenbanken }\end{array}$ & 96 & 1 & 7 & 36 \\
\hline
\end{tabular}

Tab. 15

Dabei haben die folgenden Produkte die größte Marktdurchdringung [7]:

\begin{tabular}{|l|l|}
\hline Chemie/Pharmazie & $\begin{array}{l}\text { WEMA (FIZ Technik); BEILSTEIN Crossfire; Ulimann's Encyclopedia } \\
\text { of Industrial Chemistry; Chemical Abstracts (CA/CAS); Perinorm }\end{array}$ \\
\hline Elektrotechnik & $\begin{array}{l}\text { ZDEE-FIZ Technik; INSPEC; FIZ Technik Elektrotechnik, Elektronik; } \\
\text { COMPENDIX; TEMA - Technik und Manadement }\end{array}$ \\
\hline Geschichte & WISO III; Periodical Contents Index; \\
\hline Mathematik & MATH; MathDi-ZDM; MathSciNet \\
\hline Psychologie & PSYINDEX, PsyCInfo \\
\hline
\end{tabular}

Tab. 16: Marktdurchdringung

$\mathrm{Zu}$ den fachübergreifenden Datenbanken werden die Produkte des Institute of Scientific Information (ISI) mit den Datenbanken Current Contents Connect, Journal Citation Reports, Science Citation Index, Social Science Citation Index und Arts and Humanities Citation Index gezählt. Darüber hinaus fal- len folgende Datenbanken in diese Kategorie: Internationale Bibliographie der Rezensionen, die Internationale Bibliographie der Zeitschriftenliteratur und die Datenbank World Affairs Online.

Die Verteilung auf die Bibliothekstypen verdeutlicht, dass auch hier Universitätsbibliotheken als Abnehmer fach- 
übergreifender Datenbanken eine vorrangige Position einnehmen.

\begin{tabular}{|l|c|c|c|c|}
\hline \multicolumn{1}{|c|}{ Bibliothekstyp } & UB & NB & RB & FHB \\
\hline $\begin{array}{l}\text { Anzahl der beziehenden } \\
\text { Bibliotheken }\end{array}$ & 25 & 1 & 2 & 6 \\
\hline $\begin{array}{l}\text { Anzahl der fachuber- } \\
\text { greifenden Datenbanken }\end{array}$ & 91 & 7 & 12 & 18 \\
\hline $\begin{array}{l}\text { Anteil der facherübergreif- } \\
\text { enden Datenbanken am } \\
\text { Gesamtumfang in \% }(n=128)\end{array}$ & $71 \%$ & $6 \%$ & $9 \%$ & $14 \%$ \\
\hline
\end{tabular}

Tab. 17: fachübergreifende Datenbanken/Bibliothekstyp

Dabei entfallen auf die Datenbanken des Institute of Scientific Information (ISI):

\begin{tabular}{|c|c|c|c|c|c|}
\hline Bibliothekstyp & $\begin{array}{c}\text { Science } \\
\text { Citation } \\
\text { Index }\end{array}$ & $\begin{array}{c}\text { Social } \\
\text { Science } \\
\text { Citation } \\
\text { Index }\end{array}$ & $\begin{array}{c}\text { Current } \\
\text { Contents } \\
\text { Connect }\end{array}$ & $\begin{array}{c}\text { Journal } \\
\text { Citation } \\
\text { Reports }\end{array}$ & $\begin{array}{c}\text { Arts and } \\
\text { Humanities } \\
\text { Citation } \\
\text { Index }\end{array}$ \\
\hline UB & 25 & 12 & 10 & 10 & 13 \\
\hline NB & 1 & 1 & 1 & 1 & 1 \\
\hline LB & 1 & 1 & 1 & $\cdots$ & 1 \\
\hline FHB & 3 & 2 & 2 & & 2 \\
\hline
\end{tabular}

Tab. 18: Datenbanken des Institute of Scientific Information

\section{Zusammenfas- sung und Bewer- tung der Ergebnisse}

Universitätsbibliotheken sind die hauptsächlichen Abnehinformation. Dies gilt für die elektronischen Zeitschriften wie auch für die Datenbankprodukte. Es werden überwiegend kostenpflichtige Produkte von kommerziellen Anbietern bezogen. Es sind insbesondere die Universitätsbibliotheken, die die elektronischen Zeitschriften mit einem hohen Impact Faktor bereitstellen. Es sind dies vor allem Fachzeitschriften aus dem Gebiet der Chemie/Pharmazie und der Psychologie. Darüber hinaus bieten die Universitätsbibliotheken den größten Anteil an Datenbanken an. Auch hier werden vor allem Datenbanken des Fachgebietes Chemie/Pharmazie lizenziert.
Die Lizenzierung fachübergreifender Datenbanken erfolgt insbesondere durch die

Universitätsbibliotheken. Dabei spielen die Produkte des ISI eine zentrale Rolle. Diese Produkte, mit deren Hilfe sich der Impact Faktor für verschiedene Disziplinen und

Zeitschriften ermitteln lässt, sind für die WissenschaftlerInnen von zentraler Bedeutung. Die Bibliotheken beziehen die im Web of Science erfassten Produkte der kommerziellen Verlage und stellen gleichzeitig die Nachweisinstrumente für die $\mathrm{Be}$ deutung dieser Zeitschriften bereit.

\section{Nutzung elektro- nischer Fachinfor- mation [8]}

\subsection{Relative Nutzungsstruk-} tur

In einer Datenbank wurden 1.750.377 Nutzungsfälle [9] erfasst, die sich auf die oben beschriebenen Fachgebiete beziehen. Die relative Nutzungsstruktur über verschiedene Verlage zeigt, dass $50 \%$ der Volltextzugriffe auf $7 \%$ der Titel entfallen. $80 \%$ der Zugriffe werden durch $25 \%$ der Titel abgedeckt. Mit Hilfe des Gini-Koeffizienten wurde für vier Verlage die Nutzung ermittelt [10].

\begin{tabular}{|l|l|l|}
\hline Verlag & Gini-Koeffizient & Statistische Grundlage \\
\hline Springer & 0,686 & 69 Bibliotheken \\
\hline Academic Press & 0,783 & 21 Bibliotheken \\
\hline Elsevier & 0,741 & 11 Bibliotheken \\
\hline Institute of Physics & 0,727 & 11 Bibliotheken \\
\hline
\end{tabular}

Tab. 19: Gini-Koeffizient mer der elektronischen Fach-

Die Verteilung ist bei allen betrachteten Verlagen ähnlich gelagert. Der Gini-Koeffizient liegt immer um 0,7 . Dies bedeutet, dass für alle Verlage eine starke Ungleichverteilung der Nutzung beobachtet werden kann. Die Strukturanalyse bezieht sich auf relative Größen und lässt daher keine quantitativen Aussagen über die absolute Nutzung zu.

\subsection{Durchschnittliche Nutzungshäufigkeit}

In einer weiteren Untersuchung wird die absolute Nutzung der Titel mit Hilfe einer festgelegten Nutzungsgrenze untersucht. Hier werden die Grenzen 12 Zugriffe/Bibliothek und 120 Zugriffe/Bibliothek/Jahr verwendet.

\begin{tabular}{|c|c|c|}
\hline Verlag & Anteil <12 & Anteil < 120 \\
\hline Academic Press & $63 \%$ & $97 \%$ \\
\hline Elsevier & $39 \%$ & $91 \%$ \\
\hline Springer & $48 \%$ & $93 \%$ \\
\hline Institute of Physics & $21 \%$ & $83 \%$ \\
\hline
\end{tabular}

Tab. 20: Zugriffe

$63 \%$ der Titel von Academic Press werden unter 12Mal/ Jahr und Bibliothek im Durchschnitt abgerufen. 97\% werden weniger als $120 \mathrm{Mal} / \mathrm{Jahr}$ im Volltext nachgefragt. Daraus lässt sich ableiten, dass ein Grossteil der Titel von Academic Press sehr wenig genutzt wird. Das Institute of Physics weist eine niedrigere Quote auf, was auf eine relativ höhere Nutzung hinweist.

Für die Fachgebiete Chemie/Pharmazie, Elektrotechnik, Mathematik und Psychologie wurden die absoluten Nutzungshäufigkeiten untersucht. Blackwell Science und ACS wurden aufgrund der geringen Datengrundlage nicht berücksichtigt.

Chemie/Pharmazie

\begin{tabular}{|l|l|l|l|}
\hline & \multicolumn{1}{|c|}{ Gesamtliste } & \multicolumn{1}{|c|}{ Anteił < 12 } & \multicolumn{1}{|c|}{ Anteil < 120 } \\
\hline EZB-Angaben & 1034 & & - \\
\hline Erfasste Journals & $280(27,1 \%)$ & $77(27,5 \%)$ & $246(87,9 \%)$ \\
\hline Anteil Springer & $12,5 \%$ & $11,7 \%$ & $13,4 \%$ \\
\hline Anteil Academic Press & $10,4 \%$ & $19,5 \%$ & $10,2 \%$ \\
\hline Anteil Elsevier & $76,8 \%$ & $68,8 \%$ & $76 \%$ \\
\hline
\end{tabular}

Tab. 21: Zugriffe Chemie/Pharmazie 
- Angebots-, Nutzungs- und Bezugsstrukturen elektronischer Fachinformation in Deutschland
Die elektronische Zeitschriftenbibliothek listet im Gebiet Chemie/Pharmazie insgesamt 1034 Titel, von denen 280 Titel $(27,1 \%)$ auch in der Nutzungsdatenbank erfasst sind. Von diesen Titeln sind $12,5 \%$ von Springer, $10,4 \%$ von Academic Press und $76,8 \%$ von Elsevier. 77 Titel $(27,5 \%)$ werden im Jahresdurchschnitt weniger als 12 Mal pro Bibliothek genutzt. Von diesen gering genutzten Titeln sind 11,7\% Titel vom Springer Verlag, 19,5\% Titel von Academic Press und $68,8 \%$ Titel von Elsevier. Man kann also erkennen, dass Academic Press überproportional viele gering genutzte Titel in diesem Fachgebiet aufweist.

\section{Elektrotechnik}

Im Fachgebiet Elektrotechnik liegt die Quote der mit Nutzungszahlen erfassten Titel bei $18,5 \%$. Titel von Springer und Academic Press weisen eine überproportional geringe Nutzung (unter 12 pro Jahr) auf (Tab. 22).

Mathematik

Die Tabelle zeigt die relative Nutzungssituation im Fachgebiet Mathematik. Hier liegt die Quote der erfassten Zeit-

\begin{tabular}{|l|l|l|l|}
\hline & \multicolumn{1}{|c|}{ Gosamtliste } & \multicolumn{1}{c|}{ Anteil < 12 } & \multicolumn{1}{c|}{ Anteil < 120 } \\
\hline EZB-Angaben & 411 & & - \\
\hline Erfasste Journals & $76(18,5 \%)$ & $27(35,3 \%)$ & $68(89,5 \%)$ \\
\hline Anteil Springer & $11,8 \%$ & $22,2 \%$ & $13,2 \%$ \\
\hline $\begin{array}{l}\text { Anteil Academic } \\
\text { Press }\end{array}$ & $5,3 \%$ & $11,1 \%$ & $5,9 \%$ \\
\hline Anteil Elsevier & $75 \%$ & $63 \%$ & $72,1 \%$ \\
\hline
\end{tabular}

Tab. 22: Zugriffe Elektrotechnik

\begin{tabular}{|l|l|l|l|}
\hline & \multicolumn{1}{|c|}{ Gesamtliste } & \multicolumn{1}{|c|}{ Anteil < 12 } & \multicolumn{1}{|c|}{ Anteil < 120 } \\
\hline EZB-Angaben & 601 & - & - \\
\hline Erfasste Journals & $118(19,6 \%)$ & $75(63,6 \%)$ & $114(96,6 \%)$ \\
\hline Anteil Springer & $42,4 \%$ & $41,3 \%$ & $43,9 \%$ \\
\hline Anteil Academic Press & $17 \%$ & $24 \%$ & $17,5 \%$ \\
\hline Anteil Elsevier & $37,8 \%$ & $34,7 \%$ & $38,6 \%$ \\
\hline
\end{tabular}

Tab. 23: Zugriffe Mathematik

\begin{tabular}{|l|l|l|l|}
\hline & \multicolumn{1}{|c|}{ Gesamtliste } & \multicolumn{1}{|c|}{ Anteil < 12 } & \multicolumn{1}{|c|}{ Anteil < 120 } \\
\hline EZB-Angaben & 1101 & & - \\
\hline Erfasste Journals & $97(8,8 \%)$ & $33(34 \%)$ & $88(90,7 \%)$ \\
\hline Anteil Springer & $19,6 \%$ & $15,2 \%$ & $17,1 \%$ \\
\hline Anteil Academic Press & $20,6 \%$ & $12,1 \%$ & $22,7 \%$ \\
\hline Anteil Elsevier & $59,8 \%$ & $72,7 \%$ & $60,2 \%$ \\
\hline
\end{tabular}

Tab. 24: Zugriffe Psychologie

schriften bei $19,6 \%$. Auffallend ist hierbei, dass die Titel von Academic Press überproportional wenig genutzt werden (Tab. 23).

Psychologie

Im Fachgebiet Psychologie liegt die Quote der mit Nutzungsangaben erfassten Titel mit $8,8 \%$ am niedrigsten im Vergleich zu den anderen Fachgebieten. Hier ist Elsevier überproportional stark bei gering genutzten Titeln vertreten (Tab. 24).

Zusammenfassend kann man sagen, dass insbesondere Titel von Academic Press in den meisten Fachgebieten gering genutzt werden. Für die einzelnen Fachgebiete wurden folgende Gini-Koeffizienten ermittelt:

\begin{tabular}{|l|c|}
\hline \multicolumn{1}{|c|}{ Fachgebiet } & Gini-Koeffizient \\
\hline Chemie u. Pharmazie & 0,764 \\
\hline Elektrotechnik & 0,757 \\
\hline Mathematik & 0,755 \\
\hline Psychologie & 0,815 \\
\hline
\end{tabular}

erfassten Zeitschriften $8,8 \%$ keine sicheren Aussagen zulässt

\subsection{Korrelation zwischen Nutzungshäufigkeit und Höhe des Impact Faktor} Im folgenden Abschnitt wird analysiert, ob die Nutzungsintensität mit der Höhe des Impact Faktor oder der Höhe der Total Cites 2000 korreliert. Hierzu mussten zunächst zu der EZB-Einteilung passende Klassifizierungen im Web of Science gefunden werden.

Für die Korrelation mit dem Impact Faktor ergibt sich folgender Zusammenhang (Tab. 26):

Insgesamt kann bis auf das Fachgebiet Chemie/Pharmazie eine geringe Korrelation festgestellt werden. Es ist daher zu vermuten, dass kein nennenswerter Zusammenhang zwischen den beiden Größen besteht. Die Auswertung bezüglich der Korrelation zwi-

\section{Tab. 25: Gini-Koeffizient}

Bei dieser Untersuchung stellt sich heraus, dass eine starke Ungleichverteilung in den betrachteten Fachgebieten vorliegt. Im Gebiet der Psychologie ist der Gini-Koeffizient erhöht, wobei hier die statistische Grundlage (Quote der

\begin{tabular}{|l|r|l|}
\hline \multicolumn{1}{|c|}{ Fachgebiet } & Korrelation ${ }^{11}$ & Interp retation \\
\hline Top $10 \%$ & $+0,147$ & schwach positiv \\
\hline Organic Chemistry & $+0,745$ & mittel positiv \\
\hline Elec. engineering & $+0,028$ & schwach positiv \\
\hline History & $-0,4$ & $\begin{array}{l}\text { schwach } \\
\text { negativ }\end{array}$ \\
\hline Mathematics & $+0,126$ & schwach positiv \\
\hline Psychology & $+0,321$ & schwach positiv \\
\hline
\end{tabular}

Tab. 26: Korrelation Impact Faktor/ Total Cites

\begin{tabular}{|l|r|l|}
\hline \multicolumn{1}{|c|}{ Fachgebiet } & Korrelation & Interpretation \\
\hline Top $10 \%$ & $+0,382$ & schwach positiv \\
\hline Organic Chemistry & $+0,755$ & mittel positiv \\
\hline Elec, engineering & $+0,498$ & schwach positiv \\
\hline History & $-0,2$ & schwach negativ \\
\hline Mathematics & $+0,452$ & schwach positiv \\
\hline Psychology & $+0,643$ & mittel positiv \\
\hline
\end{tabular}

Tab. 27: Korrelation Nutzungsintensität/ Total Cites schen der Nutzungsintensität und den Total Cites 2000 lässt sich wie folgt zusammenfassen (Tab. 27):

Auch die Korrelation zwischen der Nutzungsintensität und den Total Cites 2000 zeigt keine positive oder negative Tendenz.

Die Ergebnisse lassen den Schluss zu, dass kein starker Zusammenhang zwischen der Häufigkeit der Nutzung eines Titels und der Höhe des Impact Faktors bzw. den Total Cites 2000 besteht.

7.4 Durchschnittliche Nutzungshäufigkeiten nach Bibliothekstypen und Verlag gegliedert Es soll nun unter. sucht werden, wie sich die durchschnittliche Jahresnutzung innerhalb verschiedener Bib. 
liothekstypen in den relevanten Fachgebieten verhält. Dabei soll wiederum nach den erfassten Verlagen differenziert werden. Es werden Universitäts-, FachhochschulRegional- und Spezialbibliotheken unterschieden. Wiederum wird die durchschnittliche Titelnutzung pro Bibliothek pro Jahr betrachtet. Für Springer ergibt sich folgendes Bild (Grafik. 28):

Bei Universitätsbibliotheken werden die Titel von Springer insbesondere in den Bereichen Psychologie und Chemie/Pharmazie stark genutzt. Im Vergleich zwischen den Bibliothekstypen fällt auf, dass die Nutzung an Universitätsbibliotheken am meisten ausgeprägt ist. Spezialund Fachhochschulbibliotheken fallen hier deutlich zurück. Auffallend ist hier auch die relativ starke Nutzung bei Regionalbibliotheken.

Die durchschnittliche Nutzung von Academic Press Titeln ist insbesondere in den Bereichen Chemie/Pharmazie und Psychologie an Universitäts- und Spezialbibliotheken ausgeprägt. In den Bereichen Mathematik und Elektrotechnik sind nur geringe durchschnittliche Zugriffe festzustellen. Auch hier liegen die Fachhochschulbibliotheken in den durchschnittlichen $\mathrm{Zu}$ griffszahlen hinter den Universitäts-, Regional- und Spezialbibliotheken.

Die Fachgebiete Elektrotechnik und Chemie/Pharmazie weisen bei der Nutzung von Elsevier Titeln besonders an Universitäts- und Spezialbibliotheken einen hohen Stellenwert auf. Weiterhin wird an Universitätsbibliotheken auch im Bereich Psychologie eine hohe durchschnittliche Titelnutzung erzielt. Fachhochschulbibliotheken liegen zusammen mit den Regionalbibliotheken auf den hinteren Nutzungsrängen.

Institute of Physics bietet stark themenbezogene Titel an, daher sind hier nur Titel aus den Gebieten Mathematik, Elektrotechnik und Chemie/Pharmazie zu finden. Die höchste

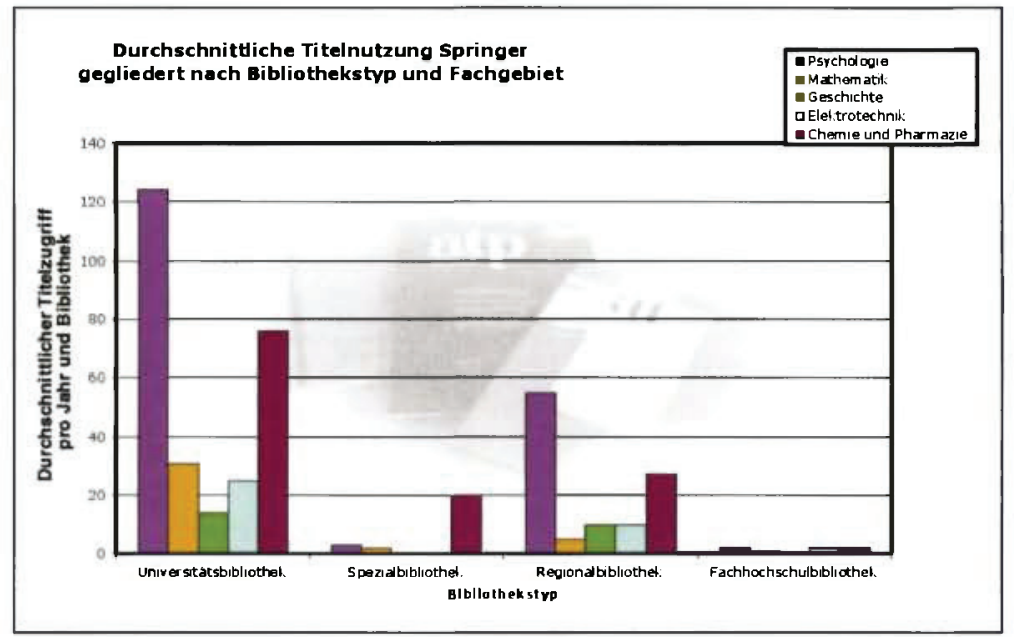

Grafik 28: Durchschnittliche Titelnutzung Springer

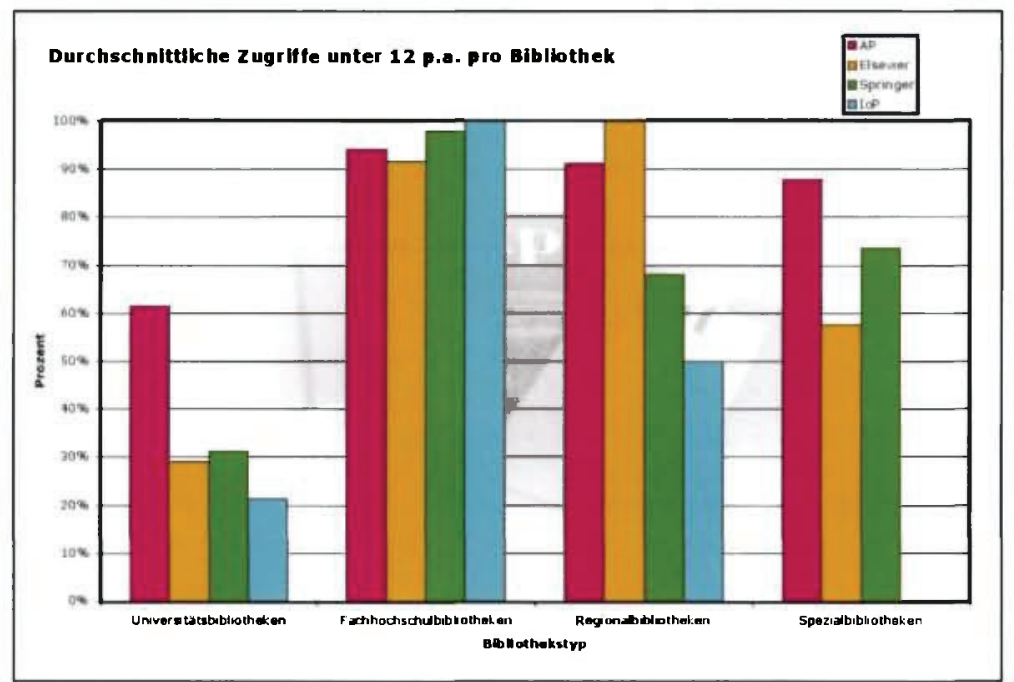

Grafik 29:

Durchschnittliche Zugriffe unter $12 /$ Jahr durchschnittliche Nutzung findet hier im Bereich Mathematik an Universitätsbibliotheken statt. Fachhochschul- und Regionalbibliotheken liegen wiederum weit hinter Universitätsbibliotheken zurück.

Zusammenfassend kann man sagen, dass die durchschnittliche Titelnutzung pro Jahr und Bibliothek insbesondere an Universitätsbibliotheken stark ausgeprägt ist. In der Chemie/Pharmazie kann über alle erfassten Verlage eine rege Titelnutzung festgestellt werden, ebenso in der Psychologie. In den Bereichen Elektrotechnik und Mathematik zeichnen sich insbesondere die Titel von Elsevier und Institute of Physics durch eine starke Titelnutzung aus.

In einer weiteren Analyse werden die Anteile der Titel, die unter 12 Mal im Durchschnitt innerhalb der Typenclusters abgerufen werden, ermittelt. (Grafik 29)

Hierbei ist insbesondere bei den Fachhochschulbibliotheken der große Anteil an Zeitschriften, die weniger häufig genutzt werden, zu beobachten. Auch wird deutlich, dass Academic Press in allen Bibliotheksclustern eine hohe Quote an gering genutzten Titeln aufweist.

Neben dem Angebot und der Nutzung elektronischer Fachinformation werden in den folgenden Kapiteln die existierenden Bezugsstrukturen näher betrachtet.

\section{Konsortiale \\ Bezugsformen elektronischer Fachinformation in Deutschland}

\subsection{Konsortien in} Deutschland

In den Empfehlungen der Hochschulrektorenkonferenz aus dem Jahr 2001 wird die Gründung von Bibliothekskonsortien zur Lizenzierung 
- AngebotsNutzungs- und Bezugsstrukturen elektronischer Fachinformation in Deutschland elektronischer Fachinformation als eine Lösung der Krise der wissenschaftlichen Informationsversorgung angesehen. Die Hochschulrektorenkonferenz identifiziert mehrere Faktoren, die für die krisenhafte Entwicklung maßgeblich sind:

- die exorbitante Verteuerung von Zeitschriften, besonders im Bereich der Naturund Ingenieurswissenschaften und der Medizin;

- die hohen Investitionskosten für das elektronische Publizieren, die von den Verlagen in der Regel auf die Kunden abgewälzt werden;

( ) die Konzentrations- und Monopolisierungstendenzen bei den Wissenschaftsverlagen;

○ die gestiegene und weiter ansteigende Nachfrage nach wissenschaftlicher Information [11].

Die Hochschulrektorenkonferenz rät zum Aufbau von Bibliothekskonsortien mit dem Ziel, eine "Marktmacht" [12] gegenüber den Verlagen zu etablieren. Dies wird jedoch als eine Aufgabe beschrieben, „... die die Möglichkeiten einer einzelnen Bibliothek bei weitem übersteigt. Die Bibliotheken haben sich daher z. T. auf Landesebene, $z$. T. länderübergreifend zu Konsortien bzw. Einkaufsverbünden (zusammenzuschließen) (...). Diese Entwicklung muss energisch vorangetrieben und auch da, wo sie noch nicht in Gang gekommen ist, in Angriff genommen werden ${ }^{a}$ [13].

Für den Bezug von Zeitschriften (in elektronischer und in gedruckter Form) und Datenbanken ist es in Deutschland zu einer Vielzahl von Konsortialgründungen auf unterschiedlichen Ebenen gekommen. Die Liste der GASCO. Arbeitsgemeinschaft weist gegenwärtig deutsche Konsortien aus. Die Situation ist jedoch unübersichtlich, was daran deutlich wird, dass auf der Liste Bundesländer, Verbünde und Forschungsinstitutionen gleichermaßen als Konsortien bezeichnet wer- den. Sie unterscheiden sich in ihrer Größe, d. h. in der Anzahl der an den Verträgen beteiligten Bibliotheken, in ihrer Rolle als Verhandlungsführer, in ihrer Organisationsform und in ihrer personellen Ausstattung jedoch erheblich voneinander. Im einzelnen handelt es sich um folgende Konsortien:

Friedrich-Althoff-Konsortium Berlin-Brandenburg (FAK)

Das FAK erhält weder für die Personalkosten noch für den Datenkauf finanzielle Mittel von den Ländern Berlin und Brandenburg. Das Konsortium hat eine eigene Rechtsform als Gesellschaft des bürgerlichen Rechts. Es unterhält eine Geschäftsstelle, in der eine Mitarbeiterin in Vollzeit beschäftigt ist. Diese wird durch den Kooperativen Bibliotheksverbund Berlin-Brandenburg finanziert.

Hessisches Bibliothekskonsortium (HeBIS-Konsortium) Die Geschäftsstelle des HeBIS-Konsortiums ist durch einen Erlass des Hessischen Ministeriums für Wissenschaft und Kunst eingerichtet worden. Das Konsortium hat keine eigene Rechtsform. Das Ministerium stellt dem Konsortium im Rahmen eines Bibliothekssonderprogramms Fördermittel zur Verfügung und beteiligt sich an den Personalkosten für die Geschäftsstelle. Die Finanzierung des Konsortiums erfolgt zu 35\% aus den Mitteln der Konsorten und zu $65 \%$ aus zentralen Mitteln.

\section{Bayern-Konsortium}

Das Bayern-Konsortium unterhält keine Geschäftsstelle, sondern organisiert das Konsortialgeschäft nebenamtlich. Die Finanzierung hierfür erfolgt aus den Eigenmitteln der Bayerischen Staatsbibliothek. Die Datenkäufe werden zu $40 \%$ aus zentralen Mitteln und zu $60 \%$ durch die Mittel der Konsorten finanziert. Personell findet eine Trennung für den Erwerb von Datenbanken und elektronischen Zeitschriften statt.
Nordrhein-Westfälisches Konsortium

Das nordrhein-westfälische Konsortium ist am Hochschulbibliothekszentrum angesiedelt. Personell findet eine Trennung zwischen dem Erwerb von Datenbanken und elektronischen Zeitschriften statt. Die Datenkäufe werden zu $50 \%$ aus zentralen Mitteln und zu 50\% aus den Mitteln der Konsorten finanziert.

\section{Sachsen}

Sachsen unterhält eine Arbeitsgemeinschaft, die die Lizenzierung der elektronischen Fachinformation vornimmt. Für den Einkauf der Daten erhält Sachsen 85\% zentrale Mittel. 15\% werden durch die neun teilnehmenden Bibliotheken selbst erbracht. Die Einkaufsgemeinschaft unterhält keine $\mathrm{Ge}$ schäftsstelle.

\section{Thüringen}

Die Arbeitsgruppe „Konsortialfragen in Thüringen “ übernimmt die Koordination und Organisation sämtlicher Aktivitäten, die im Zusammenhang mit elektronischen Datenkäufen stehen. Die Bibliotheken erhalten vom Land Thüringen weder für die Datenkäufe noch für Personalkosten zentrale Mittel.

\section{Max-Planck-Gesellschaft} (MPG)

Die Datenkäufe werden durch Mitarbeiter, die in der Generalverwaltung der MaxPlanck-Gesellschaft beschäftigt sind, organisiert und abgewickelt. Die einzelnen Institute beteiligen sich mit einem festgelegten Prozentsatz an der Finanzierung der elektronischen Fachinformation.

\section{Niedersachsen/GBV}

Die Lizenzierung elektronischer Fachinformation in Niedersachsen bzw. auf der Ebene des Gemeinsamen Bibliotheksverbundes erfolgt nicht in festgelegten Strukturen. Eine Geschäftsstelle existiert nicht. Es erfolgen Lizenzierungen auf Länderebene als auch über den GBV. Über die 


\begin{tabular}{|c|c|}
\hline Bundesland bzw. Institution & Höhe der zentralen Mittel in Euro \\
\hline Baden-Württemberg & ca. 1.290 .000 \\
\hline Bayern & ca. 1.100 .000 \\
\hline Berlin, Brandenburg & keine zentralen Mittel \\
\hline Bremen & ca. 500.000 \\
\hline Hamburg & keine Ang aben. \\
\hline Hessen & ca. 1.000 .000 \\
\hline Mecklenburg-Vorpommern & keine zentralen Mittel \\
\hline Niedersachsen & ca. 400.000 \\
\hline Nordrhein-Westfalen & ca. $1.650 .000^{16}$ \\
\hline Rheinland-Pfalz & ca. 50.000 \\
\hline Sachsen & keine zentralen Mittel \\
\hline Sachsen-Anhalt & ca. 85.000 \\
\hline Schleswig-Holstein & keine Ang aben \\
\hline Thüringen & keine zentralen Mittel \\
\hline Max-Planck-Gesellschaft & ca. 2.000 .000 \\
\hline Fraunhofer Gesellschaft & keine Angaben \\
\hline
\end{tabular}

Tab. 30: Zentralmittel der Konsortien

Finanzierungsstruktur ist keine Aussage möglich.

Baden-Württemberg

Das baden-württembergische Konsortium unterhält keine Geschäftsstelle. Die zentrale Koordination liegt in der Verantwortung des jeweiligen Vorsitzenden. Die Verhandlungsführungen werden dezentral geführt, die Entscheidungen gemeinsam gefällt. Eine zentrale Finanzierung erfolgt für die Grundversorgung mit Datenbankprodukten. Daneben existieren zahlreiche Einkaufsgemeinschaften für Datenbanken und elektronische Zeitschriften, die durch die Konsorten finanziert werden.

\section{Andere}

Bremen, Mecklenburg-Vorpommern, Sachsen und Rheinland-Pfalz unterhalten keine Geschäftsstellen. Sie beteiligen sich überwiegend an Verträgen, die durch die Konsortien abgeschlossen werden bzw. schliessen auf lokaler oder regionaler Ebene Verträge ab.

Für die Lizenzierung elektronischer Fachinformatio stehen den Konsortien derzeit Zentralmittel in folgender Höhe zur Verfügung (Tab. 30) [15]:

Hervorgegangen sind die Konsortien aus den bestehenden Leihverkehrsregionen/ Katalogisierungsverbünden ner Einzelperson einer beteiligten Bibliothek wird ein Vertrag über den Erwerb bzw. die Nutzung elektronischer Fachinformation für Bibliotheken abgeschlossen, der über die eigene Leihverkehrsregion hinausgeht. Anbieter haben mit mehreren überregionalen Konsortien Verträge abgeschlossen. Die Lizenzierung elektronischer Fachinformation auf überregionaler Ebene setzt den Aufbau neuer Kooperationsstrukturen zwischen den beteiligten Vertragspartnern voraus.

d. bundesweite Ebene:

Unter der Verhandlungsführung einer Arbeitsgemeinschaft, einer Geschäftsstelle, einer Einzelperson einer Mitgliedsinstitution wird ein Vertrag über den Erwerb bzw. die Nutzung elektronischer Fachinformation für Bibliotheken auf bundesweiter Ebene abgeschlossen. Neben diesem Vertrag existieren in der Bundesrepublik keine weiteren Verträge mit dem gleichen Anbieter. Interessierte Bibliotheken schliessen sich dem bestehenden Vertrag an Auch für den Abschluss bundesweiter Lizenzen ist der Aufbau neuer Kooperationsstrukturen notwendig.

b. regionale Ebene:

Unter der Verhandlungsführung einer Arbeitsgemeinschaft, einer Geschäftsstelle, einer Einzelperson einer Mitgliedsinstitution wird ein Vertrag für Bibliotheken der Leihverkehrsregion bzw. der Verbundregion abgeschlossen. Verlage und Datenbankanbieter haben Vertragsabschlüsse mit zahlreichen Konsortien auf regionaler Ebene. Die regionalen Konsortien knüpfen an bestehende Kooperations- und Verwaltungsstrukturen an, wodurch der administrative Aufwand bei der Lizenzierung reduziert wird.

c. überregionale Ebene:

Unter der Verhandlungsführung einer Arbeitsgemeinschaft, einer Geschäftsstelle, einer Mitgliedsinstitution, ei-

\section{e. GASCO-Ebene:}

Die GASCO hat das Ziel, Absprachen über die Lizenzierung zwischen den beteiligten deutschsprachigen Bibliotheken zu verbessern. Zur Zeit werden durch die GASCO noch keine Lizenzierungen vorgenommen. Für den Erwerb elektronischer Fachinformation auf der GASCO-Ebene ist der Aufbau entsprechender Strukturen in Form einer Geschäftsstelle geplant [17].

Die derzeit existierenden Verträge mit Anbietern elektronischer Zeitschriften und Datenbanken lassen sich den genannten Ebenen wie folgt zuordnen

\section{a. regionale Ebene}

- für den Bezug elektronischer Zeitschriften (Tab. 31)
Angebots-, Nutzungs- und Bezugsstrukturen elektronischer

Fachinformation in Deutschland 


\begin{tabular}{|c|c|c|}
\hline Konsortium & $\begin{array}{l}\text { Gesamtanzahl der Verträge } \\
\text { (inklusive überregionale, } \\
\text { bundesweite Beteiligungen } \\
\text { und Verträge auf GASco- } \\
\text { Ebene) }\end{array}$ & $\begin{array}{l}\text { Konsortialverträge auf } \\
\text { regionaler Ebene }\end{array}$ \\
\hline Baden-Wurttemberg & 5 & 0 \\
\hline Bayem-Kon sortlum & 7 & 3 \\
\hline Bremen & 9 & 3 \\
\hline Friedrich-Althoff-Konsorttum & 9 & 4 \\
\hline Fraunhofer Gesellschaft & 3 & 2 \\
\hline Gemeinsamer Bibllotheksverbund & $\overline{0}$ & $\overline{0}$ \\
\hline Hessisches Bibliothekskonsortium & 15 & 11 \\
\hline Max-Planck-Gesel|schaft & 11 & 8 \\
\hline Mecklenburg-Vorpommern & 2 & 0 \\
\hline Niedersachsen & 4 & 1 \\
\hline Nordrheln-Westfalen & 9 & 5 \\
\hline Rheinland-Pfalz & 11 & 11 \\
\hline Saarland & 6 & 1 \\
\hline Sachsen & 5 & 1 \\
\hline Sachsen-Anhalt & 5 & 1 \\
\hline Thüringen & 8 & 2 \\
\hline Summe & 109 & $5 \mathbf{5 3}$ \\
\hline \multicolumn{2}{|c|}{$\begin{array}{l}\text { durchschnittliche Anzahl der verträge auf regionaler Ebene } \\
\text { in } \%\end{array}$} & $49 \%$ \\
\hline
\end{tabular}

\begin{tabular}{|c|c|c|c|}
\hline Konsortium & $\begin{array}{l}\text { Gesamtanzahl der } \\
\text { Verträge (inklusive } \\
\text { Abschlüsse auf } \\
\text { uberregionaler und } \\
\text { GASCO-Ebene) }\end{array}$ & $\begin{array}{c}\text { Konsortialverträge } \\
\text { auf uberregionaler } \\
\text { Ebene }\end{array}$ & $\begin{array}{l}\text { Verhandlungs fuhrung } \\
\text { und Vertragsabschluss } \\
\text { uber anderes } \\
\text { Konsortium, und zwar: }\end{array}$ \\
\hline Baden-Württemberg & 5 & 2 & Bayem \\
\hline Bayern & 7 & 3 & $\begin{array}{l}\text { mit Baden-Württemberg, } \\
\text { Sach sen }\end{array}$ \\
\hline Bremen & 9 & 4 & $\begin{array}{l}\text { FAK, Bayem, } \\
\text { HeBIS }\end{array}$ \\
\hline Friedrich-Althoff-Konsortum & 9 & 3 & Bayem, HGF \\
\hline Fraunhofer Gesellschaft & 3 & 1 & FAK \\
\hline $\begin{array}{c}\text { Gemelnsamer } \\
\text { Bibliotheksverbund }\end{array}$ & 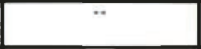 & + & -" \\
\hline $\begin{array}{c}\text { Hessisches } \\
\text { Biblioth ekskonsortlum }\end{array}$ & 15 & 2 & FAK, Bayern \\
\hline Max-Planck-Gesellschaft & 11 & 1 & FAK \\
\hline Mecklenburg-Vorpommern & 2 & + & - \\
\hline Nledersachsen & 4 & 2 & FAK \\
\hline Nordrhein-Westfalen & 9 & 3 & FAK \\
\hline Rheinland-Pfalz & 11 & -. & -" \\
\hline Saarland & 6 & 4 & NRW, FAK \\
\hline Sachsen & 5 & 3 & Bayem \\
\hline Sachsen-Anhalt & 5 & 2 & FAK \\
\hline Thüringen & 8 & 3 & FAK \\
\hline Summe & 109 & 33 & \\
\hline \multicolumn{2}{|c|}{$\begin{array}{l}\text { durchschnittliche Anzahl der Vertragge auf } \\
\text { Oberregionaler Ebene }\end{array}$} & $30 \%$ & \\
\hline
\end{tabular}

Tab. 31: Bezug elektronischer Zeitschriften regional

\begin{tabular}{|c|c|c|}
\hline Konsortium & $\begin{array}{c}\text { Gesamtanzahl der Verträge } \\
\text { (inklusive Abschlusse auf } \\
\text { uberregionaler und GASCO- } \\
\text { Ebene) }\end{array}$ & $\begin{array}{l}\text { Konsortialverträge auf } \\
\text { regionaler Ebene }\end{array}$ \\
\hline Baden-Württemberg & 28 & 23 \\
\hline Bayem-Konsortium & 25 & 21 \\
\hline Bremen & 25 & 21 \\
\hline Friedrch-Althoff-Konsortlum & 8 & 2 \\
\hline Fraunhofer Gesellschaft & 3 & 3 \\
\hline $\begin{array}{c}\text { Gemelnsamer } \\
\text { Bibliotheks verbund }\end{array}$ & 22 & 22 \\
\hline $\begin{array}{l}\text { Hessisches } \\
\text { Bibliothekskonsortium }\end{array}$ & 39 & 34 \\
\hline Max-Planck-Gesellschaft & 20 & 17 \\
\hline Mecklenburg-Vorpommern & 10 & 4 \\
\hline Niedersachsen & 19 & 15 \\
\hline Nordrheln-Westfalen & 41 & 35 \\
\hline Rheinland-Pfalz & 23 & 18 \\
\hline Sariland & 11 & 5 \\
\hline Sachsen & 13 & 9 \\
\hline Sachsen-Anhalt & 6 & 2 \\
\hline Thüringen & 27 & 21 \\
\hline Summe & 320 & 253 \\
\hline \multicolumn{2}{|c|}{ durchschnittliche Anzahl der Vertrage auf regionaler Ebene } & $79 \%$ \\
\hline
\end{tabular}

Tab. 32: Bezug Datenbanken regional

๑ für den Bezug von Datenbanken (Tab. 32)

b. überregionale Ebene

( ) für den Bezug elektronischer Zeitschriften (Tab. 33)

○ für den Bezug von Datenbanken (Tab. 34)

Tab. 33: Bezug elektronischer Zeitschriften überregional

\begin{tabular}{|c|c|c|c|}
\hline Konsortium & $\begin{array}{l}\text { Gesamtanzahl } \\
\text { der Verträge }\end{array}$ & $\begin{array}{c}\text { Konsortialverträge } \\
\text { auf uberregionaler } \\
\text { Ebene: }\end{array}$ & $\begin{array}{l}\text { Verhandlungsfuhrung und } \\
\text { Vertragsabschluss über } \\
\text { anderes Konsortium, und } \\
\text { zwar: }\end{array}$ \\
\hline Baden-Württemberg & 28 & 1 & \\
\hline Bayem-Konsortium & 25 & -. & \\
\hline Bremen & 25 & 3 & $\begin{array}{c}\text { Baden-Württemberg, NRW, FAK, } \\
\text { HeBIS }\end{array}$ \\
\hline Friedrich-Althoff-Konsortium & 8 & 3 & -- \\
\hline Fraunhofer Gesellschaft & 3 & 0 & - \\
\hline $\begin{array}{c}\text { Gemeinsamer } \\
\text { Bibliotheksverbund }\end{array}$ & 22 & ". & \\
\hline $\begin{array}{c}\text { Hessisches } \\
\text { Biblotheks konsortlum }\end{array}$ & 39 & 3 & FAK, NRW \\
\hline Max-Planck-Gesellschaft & 20 & $\mathbf{0}$ & *- \\
\hline Mecklenburg-Vorpommern & 10 & 4 & \\
\hline Nledersachsen & 19 & $\overline{0}$ & - \\
\hline Nordrhein-Westralen & 41 & 4 & $-\cdot$ \\
\hline Rheinland-Pfalz & 23 & 1 & FAK, HeBIS \\
\hline Sabrland & 11 & 4 & NRW, FAK, HABS \\
\hline Sachsen & 13 & 1 & HeBIS \\
\hline Sachsen-Anhalt & 6 & 2 & FAK, HeBIS \\
\hline Thüringen & 27 & 4 & FAK, HeBIS, NRW \\
\hline Summe & 320 & 30 & \\
\hline \multicolumn{2}{|c|}{$\underset{\%}{\text { durchschnittliche Summe der Vertrage in }}$} & $10 \%$ & \\
\hline
\end{tabular}

Tab. 34: Bezug Datenbanken überregional

\begin{tabular}{|l|l|l|}
\hline \multicolumn{1}{|c|}{ Produkt } & Verhandlungsf ührer & \multicolumn{1}{|c|}{ Bibliotheken der Lander } \\
\hline ACM Digital Library & $\begin{array}{l}\text { Hesslsches } \\
\text { Bibliothelskonsortium }\end{array}$ & $\begin{array}{l}\text { Baden-Württemberg, Bremen, Hesslsches } \\
\text { Bibliothelskonsortium, Mecklenburg- } \\
\text { Vorpommen, Niedersach sen, Nordrheln. } \\
\text { Westfalen, Saarland, Sachsen, ThUringen }\end{array}$ \\
\hline
\end{tabular}

Tab. 35: elektronische Zeitschriften bundesweit

c. bundesweite Ebene

○ für den Erwerb elektronischer Zeitschriften (Tab. 35)
○ für den Erwerb von

Datenbankprodukten

(Tab. 36) 


\begin{tabular}{|c|c|c|}
\hline Produkt & Verhandlungsführer & $\begin{array}{l}\text { Bibliotheken der Lynder bzw. } \\
\text { Institutionen }\end{array}$ \\
\hline PsyCINFO (EBSCO) & Friedrich-Althoff-Konsortium & 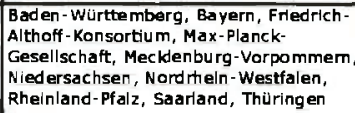 \\
\hline $\begin{array}{l}\text { SclFinder Scholar } \\
\text { (ACS,CAS) }\end{array}$ & Nordrhein-Westralen/MBZ & 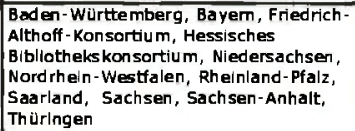 \\
\hline
\end{tabular}

Tab. 36: Datenbanken bundesweit

d. GASCO-Ebene

- für den Erwerb elektronischer Zeitschriften

\begin{tabular}{|c|c|l|}
\hline Produkt & Verhandlungsführer & Bibliotheken der Lünder bzw. Institutionen \\
\hline Nature & U8 Stuttgart & $\begin{array}{l}\text { Baden-Württemberg, Bremen, Friedrich-Althoff- } \\
\text { Konsortium, Max-Planck-Gesellschaft, } \\
\text { Rheinland-Pfalz, Sachsen-Anhalt, Thüringen }\end{array}$ \\
\hline Sclence & UB Stuttgart & $\begin{array}{l}\text { Baden-Württemberg, Max-Planck-Gesellschaft, } \\
\text { Rheinland-Pfalz, Sachsen-Anhalt, Thüring en }\end{array}$ \\
\hline
\end{tabular}

Tab. 37: GASCO elektronische Zeitschriften

Die Lizenzierung von Datenbankprodukten und elektronischen Zeitschriften erfolgt überwiegend auf regionaler Ebene unter Ausnutzung der bestehenden Kooperationsstrukturen der Verbundteilnehmer. Durchschnittlich $79 \%$ der Datenbankprodukte und $49 \%$ der elektronischen Zeitschriften werden hierüber erworben/lizenziert. Auf der überregionalen Ebene werden durchschnittlich $10 \%$ der Datenbanken und $30 \%$ der elektronischen Zeitschriften lizenziert. Bundesweite Kooperationen bzw. Einkaufskooperationen auf der GASCO-Ebene finden sich derzeit nur für vereinzelte Produkte. Der Anteil an in überregionalen Kooperationsformen erworbenen elektronischen Zeitschriften ist höher als der der Datenbanken. Es ist anzunehmen, dass hier ein Zusammenhang zwischen den Preiserhöhungen für die Lizenzierung elektronischer Zeitschriften und neuen, überregionalen Kooperationsformen besteht. Insgesamt handelt es sich jedoch um einen geringen Anteil.

Vor dem Hintergrund der Krise der wissenschaftlichen Informationsversorgung erscheint eine verbesserte $\mathrm{Ab}$ - bedarfsgerechten elektronischen Informationsangebotes von den wissenschaftlichen Bibliotheken ein erhebliches Maß an Know-how bei Verhandlungen mit Lieferanten und darüber hinaus entsprechende Kenntnisse in den Bereichen Informationstechnik und e-business erfordern (wird) und der aufwändige Betrieb eines nationalen Konsortiums nur mit Hilfe einer leistungsfähigen Organisationsstruktur realisiert werden kann [18]." Die Organisationsstruktur des Schweizer Konsortiums unterscheidet drei Ebenen und Organe [19]. 1. Lenkungsausschuss

2. Projektleitung mit angeschlossener Geschäftsstelle. 3. Expertengruppen

zu 1. Lenkungsausschuss Die Schweizerische Universitätskonferenz (SUK) bildet die Dachorganisation für das Schweizer Konsortium. Sie ist ein gemeinsames Organ des Bundes und der Kantone für universitätspolitische Angelegenheiten. Durch die SUK werden die gesamten universitären Einrìchtungen und auch die Fachhochschulen vertreten. Der Lenkungsausschuss ist der SUK unterstellt und diesem gegenüber rechenschaftspflichtig. Der Lenkungsausschuss gibt die Strategien für den Konsortialbezug und die Arbeitsschwerpunkte des nationalen Konsortiums vor. Konsortiallizenzen werden nur in Abstimmung mit dem Lenkungsausschuss abgeschlossen. Diesem gehören Direktoren verschiedener Bibliothekstypen und Sprachregionen, ein Vertreter aus der SUK, ein ausländischer Experte sowie die Leitung der Geschäftsstelle (ohne Stimmrecht) an.

Dieser Ausschuss tagt mindestens dreimal jährlich bzw. nach Bedarf öfter [20]. Zusammenfassend werden durch den Lenkungsausschuss folgende Aufgaben wahrgenommen:

- Sicherung des Informationsaustauschs mit den Projektpartnern

- Festlegung der strategi-
Angebots-, Nutzungs- und Bezugsstrukturen elektronischer

Fachinformation in Deutschland 
- Angebots-, Nutzungs- und Bezugsstrukturen elektronischer Fachinformation in Deutschland schen Ausrichtung in fachlichen Fragen

- Antragstellung an die SUK betreffend Ausführungsplan und Finanzplan

- Wahl der Projektleitung und der Mitglieder der fachspezifischen Expertengruppen

- Festlegung der Richtlinien für die Auswahl elektronischer Informationsprodukte

- Zusammenarbeit mit dem gesamtschweizerischen Koordinationsorgan der

Bibliotheken der Schweizer Hochschulen

- Aufsicht über den Projektvollzug durch die Projektleitung und durch die angegliederte Geschäftstelle ( ) Treffen der Kaufentscheidungen für Produkte mit einem Preis über 400.000

CHF/Konsortiallizenz

- Der Lenkungsausschuss erteilt im Rahmen zur Verfügung stehender Mittel besondere Aufträge an außenstehende Expertinnen oder Experten

zu 2: Projektleitung mit angeschlossener Geschäftsstelle Die Projektleitung mit angeschlossener Geschäftsstelle wird durch den Lenkungsausschuss bestimmt. Sie ist verantwortlich für den Aufbau der Geschäftsstelle, für die Anstellung des erforderlichen Personals, welches ihm administrativ unterstellt ist. Durch die Projektleitung werden die Aktivitäten der Geschäftsstelle begleitet. Sie stellt sicher, dass die strategischen Vorgaben des Lenkungsausschusses durch die Geschäftsstelle umgesetzt werden. Ausgehandelte bzw. genehmigte Lizenzverträge können durch die Projektleitung stellvertretend für die dem Konsortium angehörenden Bibliotheken unterzeichnet werden.

Die Leitung der Geschäftsstelle übernimmt die Vorbereitung der konsortialen Lizenzverträge entsprechend den strategischen Vorgaben des Lenkungsausschusses. Sie erhält ihre fachlichen Weisungen von der Präsidentin des Lenkungsausschusses.
Verbindlichkeiten bis zu 100.000 CHF können in eigener Verantwortung durch die Geschäftsstelle eingegangen werden. $\mathrm{Zu}$ den Aufgaben gehören im einzelnen:

- Aufbau und Erweiterung der Strukturen des Konsortiums

- Führen der laufenden Geschäfte (Infrastruktur des Konsortiums, Lizenzierung von elektronischer Fachinformation)

- Beratung und Unterstützung der Hochschulbibliotheken in Fragen, die elektronische Publikationen betreffen - Führen des Sekretariats des Lenkungsausschusses - Information der Hochschulbibliotheken und der Öffentlichkeit über die Aktivitäten des Konsortiums

- Evaluation bezüglich der Leistungen der Infrastruktur und der Nutzung von lizenzierten elektronischen Ressourcen

- Abrechnung der individuellen Kostenanteile der jeweiligen Partner von

konsortialen Lizenzverträgen - Zusammenarbeit mit Konsortialpartnern im In- und Ausland

- Zusammenarbeit mit den fachspezifischen Expertengruppen und Koordination der Tätigkeiten

- Zusammenarbeit mit den Infrastruktureinrichtungen vor Ort

- Zusammenarbeit mit Experten in juristischen Fragen - Organisation der inhaltlichen und finanziellen Berichterstattung

\section{zu 3. Expertengruppen}

Das Konsortium hat Expertengruppen in den Bibliotheken benannt, die über besondere Produktkenntnisse in ihren Fachgebieten verfügen und dem Lenkungsausschuss und der Geschäftsstelle beratend zur Seite stehen.

\subsubsection{Niederlande}

Das UKB-Konsortium [21] wurde bereits 1977 mit dem Ziel gegründet, den Zugang und das Angebot an wissenschaftlicher Information in den Bibliotheken der Nieder- lande zu verbessern. In dem Konsortium sind die Bibliotheken der allgemeinen Universitäten, der technischen Universitäten, die Bibliothek der agrarwissenschaftlichen Universität und die Königliche Bibliothek organisiert. Weiterhin besteht eine assoziierte Mitgliedschaft der Open University Netherlands und der Forschungsbibliothek NIWI (Niederländisches Institut für wissenschaftliche Information). Für die Fachhochschulen soll im kommenden Jahr erstmals eine Beteiligung am konsortialen Erwerb möglich werden. Das Konsortium erhält keine zentralen Mittel für den Erwerb elektronischer Fachinformation.

Die Organisationsstruktur des UKB-Konsortiums unterscheidet drei Ebenen:

1.Arbeitsgemeinschaft der Universitätsbibliotheken-UKB

2. Lizenzbüro

3. Expertengruppen in den Universitätsbibliotheken

zu 1: Arbeitsgemeinschaft der Universitätsbibliotheken Die Arbeitsgemeinschaft der niederländischen wissenschaftlichen Bibliotheken nimmt neben anderen Tätigkeiten auch die Lizenzierung elektronischer Fachinformation wahr. Sie setzt sich zusammen aus den Bibliotheksdirektoren/-direktorinnen der beteiligten Bibliotheken. Mit der Verhandlungsführung werden aus diesem Kreis 1-2 Personen betraut. Bevor die Verhandlungsführer mit einem Angebot des Konsortiums an die Verlage herantreten, wird der Bedarf an der jeweiligen Lizenz und die Bedingungen der Lizenzierung zwischen den TeilnehmerInnen abgestimmt. Auf der Basis einer Modelllizenz, die durch die Arbeitsgemeinschaft erarbeitet wurde, wird die Verhandlungsführung durchgeführt. Die Arbeitsgemeinschaft strebt lange Laufzeiten mit den Anbietern an, da dies die administrativen und die logistischen Kosten senken kann. 
zu 2: Lizenzbüro

Das UKB-Konsortium unterhält eine Geschäftsstelle, deren Personalkosten für 1,5 Stellen über das Forschungsnetz der Niederlande finanziert werden. Das Lizenzbüro hat folgende Aufgaben:

- Information über neue Lizenzangebote für die Bibliotheken

- Bedarfsermittlung in den Bibliotheken

- Aufbereitung der Interessenbekundungen der Bibliotheken

- Beantwortung von Fragen der beteiligten Bibliotheken - Einholen eines Angebotes bzw. alternativer Angebote auf der Grundlage der Interessenbekundungen

- Fertigstellung von Beschlussvorlagen auf der Basis des vorliegenden Verhandlungsangebotes

zu 3: Expertengruppen in den Universitätsbibliotheken

Die Expertengruppen haben beratenden Charakter und unterstützen die Arbeit des Lizenzbüros und der Arbeitsgemeinschaft durch Empfehlungen.

8.2.3 Bewertung dieser konsortialen Organisationsformen

In dem Schweizer und in dem niederländischen Konsortium sind die abgestuften Verantwortungsbereiche gut erkennbar. Die Funktion des Lenkungsausschusses/der Arbeitsgemeinschaft besteht in der Entwicklung strategischer Vorgaben für den Konsortialbezug. Die darunter angesiedelten Organe haben einen operativen Charakter. Von Interesse ist die abgestufte finanzielle Verantwortung der einzelnen Ebenen in dem Schweizer Konsortium. Dies entlastet den Lenkungsausschuss und bindet die Ebene des Präsidiums und der Geschäftsstelle in die finanzielle Verantwortung ein.

Die Leitung der Geschäftsstelle des Schweizer Konsortiums und des Lizenzbüros in den Niederlanden beurteilen die Möglichkeit eines festen Ansprechpartners für die be- teiligten Bibliotheken als vorteilhaft. Anfragen und Interessen der Mitgliedseinrichtungen können an zentraler Stelle gebündelt und in konzentrierter Form bearbeitet werden.

Als problematisch beurteilt die Leitung der Geschäftsstelle in der Schweiz, dass die Bedürfnisse kleinerer Institutionen in nicht ausreichender Form Berücksichtigung finden. Weiterhin wird die Kooperation zwischen den beteiligten Bibliotheken, z.B. in Fragen der Schulungen als verbesserungswürdig angesehen.

\subsection{Mögliche Aufgaben der GASCO}

Die GASCO könnte bei einer Verstärkung der überregionalen und bundesweiten Kooperation einen ausschließlich strategischen Charakter annehmen. Sie würde keine eigene Geschäftsstelle für die Lizenzierung von elektronischen Fachinformationen unterhalten. Sie sucht die Kooperationen zu Initiativen, Projekten und Gremien, die auf eine Veränderung der Wertschöpfungskette im digitalen Umfeld abzielen, unterstützt diese Aktivitäten, indem sie die Konsortien und andere Entscheidungsträger hierüber informiert. Die Veränderung der bestehenden Wertschöpfungskette und die Entwicklung neuer Geschäftsmodelle für die Lizenzierung von elektronischer Fachinformation wird damit auch durch die GASCO vorangetrieben.

Das Gremium entwickelt die Leitlinien des konsortialen Bezugs für die Bundesrepublik Deutschland, die durch die überregional bzw. bundesweit agierenden Konsortien umgesetzt werden.

Hinsichtlich der internen Organisation hat sich in der Schweiz und den Niederlanden herausgestellt, dass ein fester Ansprechpartner in den Geschäftsstellen von Vorteil ist. Werden die hierfür erforderlichen Mittel nicht zentral bereitgestellt, ist zu überlegen, ob zumindest ein Teil der Per- sonalkosten über Mitgliedsbeiträge der Konsorten finanziert werden. Der GASCO und den Geschäftsstellen der überregional arbeitenden Konsortien stehen nach fachlichen Gesichtspunkten zusammengesetzte überregional kooperierende Expertengruppen zur Verfügung. Sie informieren und beraten auch andere Bibliotheken und tragen auf diese Weise zur Entlastung der Geschäftsstellen bei.

Grundsätzlich darf bei diesen Überlegungen nicht außer acht gelassen werden, dass eine Lösung der Krise der wissenschaftlichen Informationsversorgung durch die Weiterentwicklung der bestehenden Strukturen nicht herbeigeführt wird. Das Ungleichgewicht zwischen Anbieter und $\mathrm{Ab}$ nehmer elektronischer Fachinformation lässt sich in der langfristigen Perspektive nur durch die Entwicklung eigener Publikationssysteme und den Aufbau elektronischer Archive verändern.

\section{Ergebnisse einer Befragung verschiedener. Konsortien}

\subsection{Zielsetzungen von \\ Konsortien}

Mit Hilfe eines standardisierten Fragebogens wurde die Selbsteinschätzung der Konsortien hinsichtlich verschiedener Zielsetzungen ermittelt [22]. In einem ersten Schritt wurde eine Gewichtung möglicher Zielsetzungen der Konsortien vorgenommen, wobei die Ziffer 1 das wichtigste Ziel darstellt. (Tab. 38)

Im Vordergrund steht der inhaltliche Aspekt konsortialer Tätigkeit, nämlich die „Vergrößerung des Angebotes für die Endnutzer "Dieses wird mit Abstand als wichtigste Zielsetzung angesehen. Erst dann folgen Zielsetzungen, die finanzielle Aspekte in den Vordergrund stellen. In einem zweiten Schritt wurden die Zielsetzungen mit der Selbsteinschätzung der Konsortien verglichen. 


\begin{tabular}{|c|c|c|c|c|}
\hline $\begin{array}{l}\text { Zieisetzungen des } \\
\text { Konsortiums }\end{array}$ & $\begin{array}{c}\text { Gewichtung } \\
\text { der } \\
\text { Zielsetzung }\end{array}$ & $\begin{array}{l}\text { Summe der } \\
\text { Wertungen }\end{array}$ & $\begin{array}{l}\text { Anzahl der } \\
\text { Nennungen }\end{array}$ & \begin{tabular}{|l} 
Anzahl der \\
Enthaltungen
\end{tabular} \\
\hline $\begin{array}{l}\text { Vergrößerung des Angebotes für die } \\
\text { Endnutzer }\end{array}$ & 1 & 6 & 6 & 4 \\
\hline $\begin{array}{l}\text { Reduzlerung der Erwerbungs kosten Im } \\
\text { Sinne von "Gleiche Kosten, mehr } \\
\text { Inhalte" }\end{array}$ & 2.2 & 13 & 6 & 4 \\
\hline $\begin{array}{c}\text { Redu zierung der Erwerbungskosten im } \\
\text { Sinne von "Weniger Kosten, glelche } \\
\text { Inhalbe" }\end{array}$ & 2,8 & 14 & 5 & 5 \\
\hline Erhöhung des Infor matlons wertes & 3,1 & 22 & 7 & 3 \\
\hline $\begin{array}{l}\text { Verelnfachung der } \\
\text { Verwaltungsstrukturen }\end{array}$ & 4 & 28 & 7 & 3 \\
\hline $\begin{array}{l}\text { Bessere Kooperation } z w / s c h e n \text { den } \\
\text { Informationsversorgungseinnichtungen } \\
\text { der Region }\end{array}$ & 4 & 24 & 6 & 4 \\
\hline $\begin{array}{l}\text { Bereitstellung elner zentralen } \\
\text { Infrastruktur für die Erwerbung } \\
\text { elektron|scher Ressourcen }\end{array}$ & 4,1 & 29 & 7 & 3 \\
\hline $\begin{array}{l}\text { Umstellung von Print-Ressourcen auf } \\
\text { elektronlsche Ressourcen }\end{array}$ & 4,3 & 30 & 7 & 3 \\
\hline $\begin{array}{l}\text { Bessere Kooperation zwischen den } \\
\text { fachlich verwandten Informations- } \\
\text { versorgungseinilichtungen }\end{array}$ & 4,8 & 29 & 6 & 4 \\
\hline
\end{tabular}

Tab. 38: Zielsetzungen des Konsortiums zung: Verbesserung des Informationsangebotes (Graphik 39)

Der inhaltliche Aspekt "Vergrößerung des Angebotes für die Endnutzer" wird von sieben Konsortien als teilweise realisiert bzw. als realisiert angesehen. Hier zeigt sich eine Übereinstimmung zwischen den Ansprüchen an die konsortiale Tätigkeit und der realisierten Zielsetzung. Obwohl der Aspekt "Erhöhung des Informationswertes" als
Zielsetzung keinen vorrangigen Platz einnimmt, sehen sieben der neun befragten Konsortien diese Zielsetzung als teilweise realisiert bzw. als realisiert an.

- Realisierung der Zielsetzung: Reduzierung der Erwerbungskosten (Graphik 40)

Die Reduzierung der Erwerbungskosten steht in dem Ranking der Zielsetzungen an zweiter bzw. an dritter Stelle.

Acht Konsortien sind der Auffassung, dass sich der Erwerb elektronischer Fachinformation im Sinne "Gleiche Kosten, mehr Inhalte" ganz bzw. teilweise durch konsortiale Arbeit realisieren lässt. Sechs Konsortien stimmen der Aussage ganz bzw. teilweise zu, dass durch konsortiale Bezugsformen die Reduzierung der Erwerbungskosten im Sinne "Weniger Kosten, gleiche Inhalte" realisieren lässt. Auch hier ist eine relative Übereinstimmung zwischen den Ansprüchen an konsortiale Tätigkeit und der Selbsteinschätzung der Konsortien zu beobachten.

- Realisierung der Zielset- zung: Verbesserung der Infrastruktur (Graphik 41).

Obwohl die Zielsetzungen "Vereinfachung der Verwaltungsstrukturen“ und „Bereitstellung einer zentralen Infrastruktur für die Erwerbung elektronischer Ressourcen " eine eher nachrangige Rolle spielen, werden diese von sieben bzw. sechs der befragten Konsortien als teilweise bzw. als realisiert angesehen. Es handelt sich hierbei um einen positiven Nebeneffekt konsortialer Organisation.

- Realisierung der Zielsetzung: Verbesserung der Kooperation (Graphik 42)

Kooperation zwischen den Institutionen der Informationsversorgung hat als Zielsetzung keine Priorität. Die grosse Anzahl der Enthaltungen legt den Schluss nahe, dass ihr wenig Bedeutung beigemessen wird.

\subsection{Vertragsformen und Preismodelle}

Wie oben bereits gezeigt wurde, ist eine Beteiligung an Vertragsabschlüssen über die Region hinaus möglich. Daher variiert die Anzahl der be-

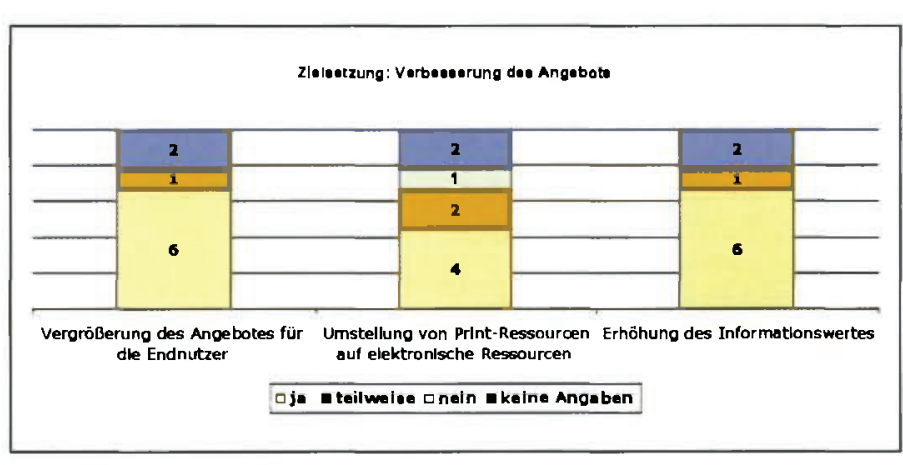

Graphik 39: Verbesserung des Informationsangebotes

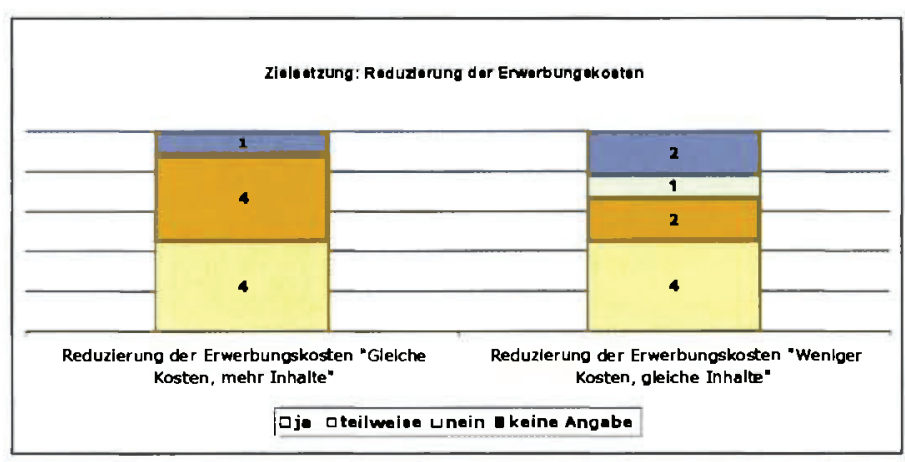

Graphik 40: Reduzierung der Erwerbungskosten

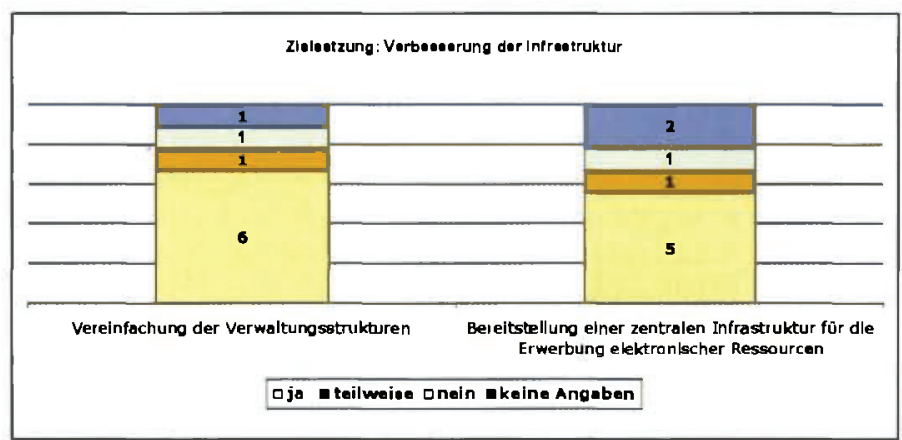

Graphik 41: Verbesserung der Infrastruktur

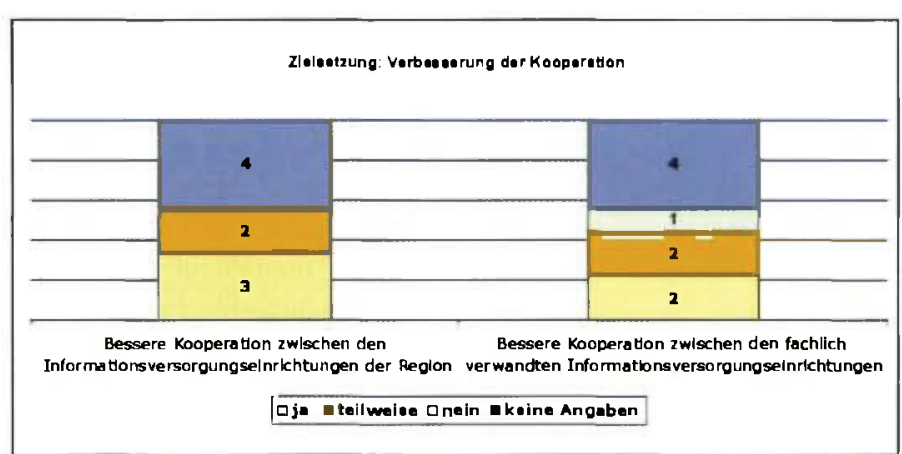

Graphik 42: Verbesserung der Kooperation 


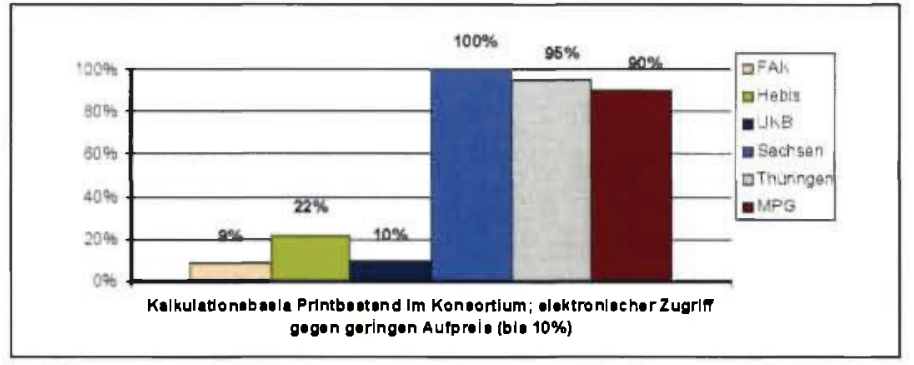

Graphik 43: Kalkulationsbasis Printbestand im Konsortium...

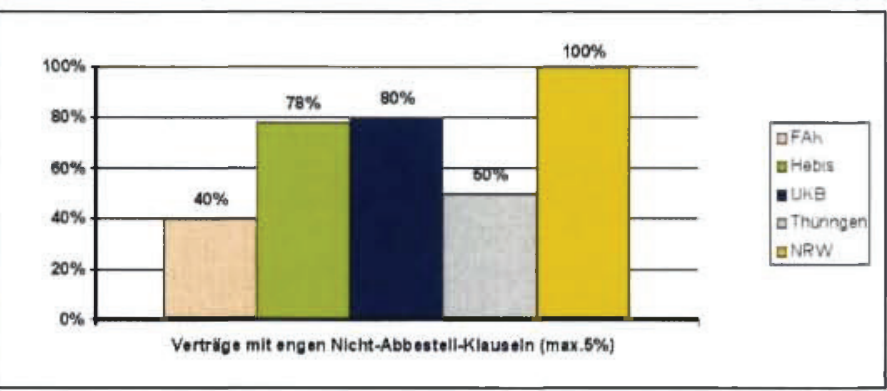

Graphik 44: Nicht-Abbestell-Klauseln

teiligten Konsorten je nach Vertrag. Im Gegensatz zu dem niederländischen Konsortium, welches eine lange Laufzeit der Verträge anstrebt, wird von sechs der neun Konsortien eine Laufzeit der Verträge von einem Jahr als wünschenswert angesehen. Derzeit überwiegen Vertragslaufzeiten von einem Jahr ( 8 von 16 Nennungen) und drei bis fünf Jahren ( 5 von 16 Nennungen).

Für die Lizenzierung elektronischer Zeitschriften und Datenbanken sind die Vertragsformen Lizenzvertrag mit campusweitem Pauschalzugriff und die Vertragsform Kaufverträge am stärksten verbreitet.

Die Preisbildung für die Lizenzierung elektronischer Zeitschriften setzt sich aus den Basiskosten für die Printexemplare und den Lizenzkosten für die elektronische Version zusammen. Der relative Anteil dieses Preismodells am Gesamtvolumen der Verträge beträgt in drei Konsortien $90 \%$ bzw. über $90 \%$ des Umsatzes. Der prozentuale Aufschlag (Lizenzkosten) für die elektronische Version der Zeitschrift variiert zwischen unter $10 \%$ und über 10\% (Graphik 43).

Bestandteil der Verträge mit den Verlagen sind Klauseln über die Höhe zulässiger Abbestellungen von Printabonnements im Vertragszeitraum. Fünf der neun Konsortien geben an, dass sie Verträge mit einer solchen Vertragsklausel abgeschlossen haben, die eine Abbestellung der Printabonnements von über 5\% verbietet [23] (Graphik 44).

Durch diese Vertragsklausel ist es den Konsortien nur sehr eingeschränkt möglich, die Kosten für den Bezug von Print- und elektronischer Zeitschrift zu reduzieren, da eine Entkoppelung der Zeitschriften in dem derzeitig dominierenden Geschäftsmodell nicht vorgesehen ist. Eine finanzielle Steuerungsmöglichkeit besteht für die Konsortien zur Zeit nur in der Ausnutzung der erlaubten $5 \%$ igen Abbestellquote und in Neuverhandlungen auf der jeweils aktuellen Grundlage des vorhandenen Printbestandes.

Die Konsortien verfolgen jedoch weitere Strategien der Kostenreduzierung, die durch veränderte Zugriffsstrukturen, dem cross access und dem additional access, realisiert werden sollen. Dieser wird gegenwärtig in folgenden Konsortien praktiziert:

\begin{tabular}{|c|c|c|c|c|}
\hline $\begin{array}{c}\text { Relativer Anteil am } \\
\text { Gesamtvolumen der Vertrage } \\
\text { in \% }\end{array}$ & FAK & Hebis & Sachsen & Thüringen \\
\hline Cross Access & $3 \%$ & $45 \%$ & $20 \%$ & $70 \%$ \\
\hline
\end{tabular}

\section{Tab. 45: Cross Access}

Die Kostenreduzierung beim Additional Access besteht hierbei in der Verteilung der Kosten auf eine größere Titelmenge und einer Reduzierung der Stückkosten. Derzeit realisiert ist der Additional Access in folgenden Konsortien [24]:

\begin{tabular}{|c|c|c|c|c|}
\hline $\begin{array}{c}\text { Relativer Anteil } \\
\text { am Gesamtvolumen der } \\
\text { Vertrage in \% }\end{array}$ & FAK & Hebis & Sachsen & Thürinaen \\
\hline Additional Access & $97 \%$ & $55 \%$ & $0 \%$ & $10 \%$ \\
\hline
\end{tabular}

Tab. 46: Additional Access

Hinsichtlich der Gestaltung der Preismodelle für elektronische Zeitschriften zeigt sich zwischen den Konsortien und den Verlagen ein deutlicher Interessenkonflikt. Dies wird durch die Diskrepanz zwischen den erwarteten und den wünschenswerten Preismodellen in der Zukunft deutlich (Tab. 47).

Mehrheitlich wird das Preismodell 12 "Mischform: Kerntitel werden pauschal lizenziert, Randtitel werden über Pay-per-View oder Kontingente abgerechnet " von den befragten Konsortien als wünschenswert angesehen. Darüber hinaus halten sie eine Aufhebung der engen Abbestellklauseln für wünschenswert.

Bei der Lizenzierung von Datenbanken überwiegt in den befragten Konsortien das Preismodell 1: Pauschaler Gesamtpreis für die (campusweite) Jahresnutzung [25].

Vier Konsortien sehen dieses Preismodell als wünschenswert an. Hier lässt sich eine gewisse Übereinstimmung der Interessen beider Akteursgruppen feststellen. Weiterhin wird das Preismodell $3{ }_{n}$ Pay-per-View (feste Preisvereinbarungen für bestimmte Nutzungsformen; bezahlt wird nur, was wirklich gebraucht wird) von vier der befragten Konsortien als 


\begin{tabular}{|c|c|c|}
\hline Preismodelle & $\begin{array}{l}\text { Erwartete } \\
\text { Entwicklung }\end{array}$ & $\begin{array}{l}\text { Wünschenswerte } \\
\text { Entwicklung }\end{array}$ \\
\hline $\begin{array}{l}\text { 1:Kalkulationsbasis Printbestand im Konsortium zu } \\
\text { gegebenem Zeitpunkt; elektronische Zugriffe } \\
\text { kostenfrei }\end{array}$ & $\ldots$ & $\cdots$ \\
\hline $\begin{array}{l}\text { 2:Kalkulationsbasis Printbestand im Konsortium; } \\
\text { elektronischer Zugriff gegen geringen Aufpreis } \\
\text { (bis } 10 \% \text { ) }\end{array}$ & $\cdots$ & $\cdots$ \\
\hline $\begin{array}{l}\text { 3:Kalkulationsbasis Printbestand im Konsortium; } \\
\text { elektronischer Zugriff gegen hohen Aufpreis } \\
\text { (über } 10 \% \text { ) }\end{array}$ & $\cdots$ & $\cdots$ \\
\hline $\begin{array}{l}\text { 4:Kalkulationsbasis elektronische "Bestände", } \\
\text { weniger als } 100 \% \text { des entsprechenden Printpreises; } \\
\text { Printversion gegen Aufpreis pauschal }\end{array}$ & 1 & 1 \\
\hline $\begin{array}{l}\text { 5:Kalkulationsbasis elektronische "Bestände" } \\
\text { weniger als } 100 \% \text { des entsprechenden Printpreises; } \\
\text { Printversion gegen Aufpreis individuell }\end{array}$ & 3 & 2 \\
\hline $\begin{array}{l}\text { 6: Kalkulationsbasis elektronische "Bestände” } 100 \% \\
\text { und mehr des entsprechenden Printpreises; } \\
\text { Printversion gegen Aufpreis }\end{array}$ & 1 & $\overline{-}$ \\
\hline $\begin{array}{l}\text { 7:Vertragsklausel: Verträge mit engen Nicht } \\
\text { Abbestell-Klauseln (max. } 5 \% \text { Abbestellung) }\end{array}$ & 1 & -- \\
\hline $\begin{array}{l}\text { 8:Vertragsklausel: Verträge mit großzügigen oder } \\
\text { ohne Nicht-Abbestell-Klauseln }\end{array}$ & -- & 4 \\
\hline $\begin{array}{l}\text { 9:Kontingentpreise (ein festes Artikelkontingent } \\
\text { wird lizenziert, ist dieses Kontingent aufgebraucht, } \\
\text { wird die nächste Lizenz vereinbart }\end{array}$ & $\cdots$ & 1 \\
\hline $\begin{array}{l}10: \text { Pay-per-View-Preise: (feste Preisverein- } \\
\text { barungen für bestimmte Nutzungsformen; bezahlt } \\
\text { wird nur das, was wirklich genutzt wird) }\end{array}$ & 2 & 2 \\
\hline $\begin{array}{l}11 \text { Pay-per-View-Preise: (feste Preisverein- } \\
\text { barungen fur bestimmte Nutzungsformen; bezahlt } \\
\text { wird nur das, was wirklich genutzt wird; die Kosten } \\
\text { werden ganz oder teilweise an die Endnutzer } \\
\text { weitergegeben) }\end{array}$ & 0 & 0 \\
\hline $\begin{array}{l}\text { 12:Mischform: Kerntitelwerden pauschal lizenziert, } \\
\text { Randtitel werden über Pay-per-View oder } \\
\text { Kontingente abgerechnet }\end{array}$ & 2 & 5 \\
\hline
\end{tabular}

Tab. 47: Geschäftsmodelle elektronische Zeitschriften

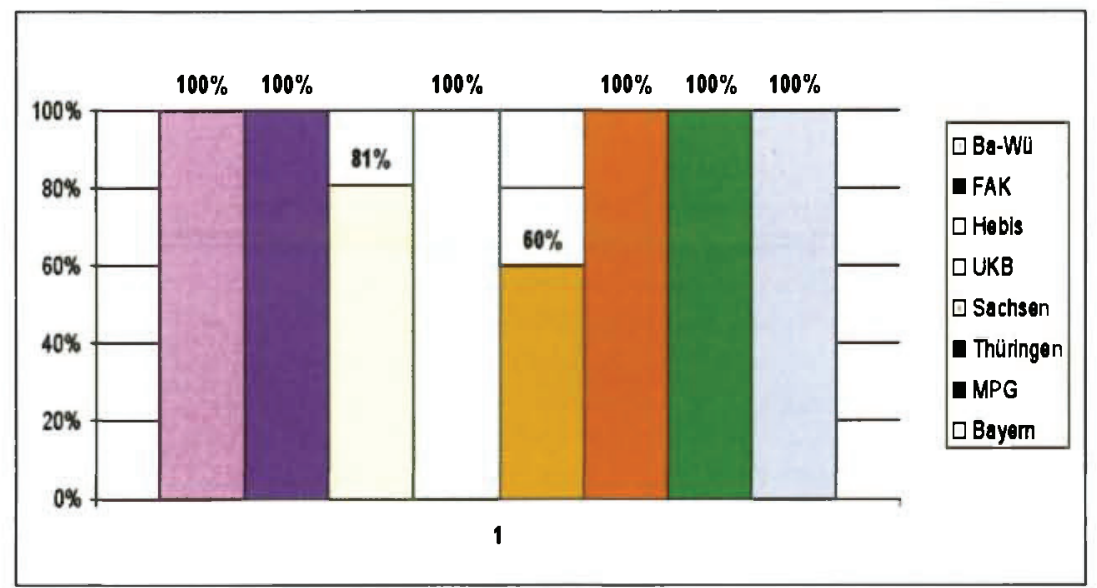

Graphik 48: Geschäftsmodell Datenbanken: pauschaler Gesamtpreis

\begin{tabular}{|l|c|c|}
\hline \multicolumn{1}{|c|}{ Preismodelle } & $\begin{array}{c}\text { Erwartete } \\
\text { Entwicklung }\end{array}$ & $\begin{array}{c}\text { Wünschenswerte } \\
\text { Entwicklung }\end{array}$ \\
\hline $\begin{array}{l}\text { 1: Pauschaler Gesamtpreis für die (campusweite) } \\
\text { Jahresnutzung }\end{array}$ & 2 & 4 \\
\hline $\begin{array}{l}\text { 2: Kontingentpreise (ein festes Zugriffskontingent } \\
\text { wird lizenziert, ist dieses Kontingent aufgebraucht, } \\
\text { wird die nächste Lizenz vereinbart }\end{array}$ & 2 & 2 \\
\hline $\begin{array}{l}\text { 3:Pay-per-View-Preise (feste Preisvereinbarungen } \\
\text { für bestimmte Nutzungsformen; bezahlt wird nur, } \\
\text { was wirklich gebraucht wird }\end{array}$ & 1 & 4 \\
\hline $\begin{array}{l}\text { 4:Pay-per-View-Preise (feste Preisvereinbarungen } \\
\text { für bestimmte Nutzungsformen; bezahlt wird nur, } \\
\text { was wirklich gebraucht wird, die Kosten werden } \\
\text { ganz oder teilweise an die Endnutzer weitergeleitet }\end{array}$ & & \\
\hline $\begin{array}{l}\text { 5: Mischform: Kernbestandteile der DB werden } \\
\text { pauschal lizenziert, Spezialteile werden über Pay } \\
\text { per-View abgerechnet }\end{array}$ & 1 & $\cdots$ \\
\hline
\end{tabular}

Tab. 49: Geschäftsmodelle Datenbanken wünschenswert angesehen, die Durchsetzung wird jedoch nicht erwartet [26] (Tab. 49).

\subsection{Preismodell: Tarifmi-} schung aus Lizenzpreis und Pay-per-View [27]

Für die Bepreisung der elektronischen Fachinformation lassen sich zwei Modelle unterscheiden. Die bereits beschriebenen Basiskosten zuzüglich Lizenzkosten für die elektronische Parallelversion einer Zeitschrift und das Pay-per-View-Verfahren. Hierbei wird jeder Zugriff auf einen Titel einzeln erfasst und mit einem Kostensatz in Rechnung gestellt. Es handelt sich hierbei um ein angebots- und bestandsorientiertes Preismodell und um ein nachfrageorientiertes Modell. Die Ergebnisse der Befragung zeigen, dass die Konsortien die Durchsetzung einer Mischung dieser beiden Tarife für wünschenswert halten. Die Lizenzierung von Kernzeitschriften und die Einzelabrechnung für Zugriffe auf Volltexte geringer genutzter Zeitschriften bietet grundsätzlich die Möglichkeit, Einsparungen zu erzielen. Als Ergebnis einer Kostenrechnung für das HeBISKonsortium (Anbieter: Elsevier) zeigt sich ein Einsparpotenzial in Höhe von $5 \%$ bis maximal 15\% [28]. Die Kosten für diese Tarifmischung stehen jedoch in enger Abhängigkeit von der Nutzung der im payper-View-Verfahren bezogenen Zeitschriftenartikel. Derzeit ist auf Anbieterseite keine Bereitschaft erkennbar, dieses Preismodell zur vertraglichen Grundlage zu machen. Eine langfristige finanzielle Entlastung der wissenschaftlichen Bibliotheken ist jedoch auch durch dieses Modell nicht zu erwarten, da davon auszugehen ist, dass die Verlage die Einzelkosten/ Zugriff entsprechend ihren Gewinnerwartungen kalkulieren werden.

9.4 Alternative Anbieter in der wissenschaftlichen Informationsversorgung Neben den kommerziellen 
Verlagen bieten Fachgesellschaften und Hochschulverlage elektronische Fachinformationen an. Die folgende Tabelle zeigt das Verhältnis von kommerziellen Verlagen, Fachgesellschaften und Hochschulverlagen in \% des Umsatzes in den befragten Konsortien:

\begin{tabular}{|c|c|c|c|c|c|c|}
\hline & Ba-Wu & FAK & HEBIS & MPG & Sachsen & Thuringen \\
\hline Hochschulverlage & $0 \%$ & $0 \%$ & $1 \%$ & $0 \%$ & $0 \%$ & $0 \%$ \\
\hline Fachgeselischaften & $0 \%$ & $20 \%$ & $1,50 \%$ & $30 \%$ & $0 \%$ & $10 \%$ \\
\hline $\begin{array}{c}\text { Kommerzielle } \\
\text { Verlage }\end{array}$ & $100 \%$ & $80 \%$ & $97,50 \%$ & $70 \%$ & $100 \%$ & $90 \%$ \\
\hline
\end{tabular}

Tab. 50: Alternative Anbieter

Für die wissenschaftliche Informationsversorgung spielen die Hochschulverlage derzeit keine Rolle. Von Interesse ist weiterhin, ob die Konsortien elektronische Ressourcen von solchen Anbietern beziehen, die sich durch wissenschaftsfreundliche Preismodelle auszeichnen.

wertes lassen sich in der Einschätzung der Konsortien durch die derzeitige Bezugsform realisieren. Den Konsortien gelingt es für die gleichen Kosten mehr Inhalte bereitzustellen. Auch die beschriebenen indirekten Rabattierungsmöglichkeiten durch Additional Access und Cross Access wirken sich noch positiv auf die Kostenentwicklung aus.

\begin{tabular}{|c|c|c|c|c|c|c|c|}
\hline & Bayern & FAK & Hebis & MPG & NRW & Sachsen & Thünngen \\
\hline Ja & $x$ & & & & & & \\
\hline Nein & & $x$ & & & $x$ & $x$ & $x$ \\
\hline Geplant & & & $x$ & $x$ & & & \\
\hline
\end{tabular}

Tab. 51: Kooperation mit alternativen Anbietern

Lediglich Bayern unterstützt solche Ansätze durch die Lizenzierung dieser Produkte. In zwei weiteren Konsortien (HeBIS, MPG) ist dies geplant. Als alternative Anbieter elektronischer Fachinformation werden BioMedCentral und SPARC genannt. Die Unterstützung dieser Initiativen und Projekte bedeutet für die Konsortien derzeit einen finanziellen Mehraufwand, da sie die "alternativen" Produkte neben den Produkten der kommerziellen Verlage lizenzieren. Auch die in direkter Konkurrenz zu hochpreisigen Zeitschriften kommerzieller Verlage heraus-gegebenen Zeitschriften von SPARC führen nicht zwangsläufig zur Abbestellung der teureren Zeitschrift, da diese in ein Titelpaket eingebettet ist und nicht als Einzelprodukt bezogen werden kann.
Die Risiken der derzeitigen Bezugsformen sind vor allem darin zu sehen, dass die Rabattierungsmöglichkeiten nicht dauerhaft zu einer positiven Kostenentwicklung beitragen. Als Indikatoren hierfür sind die Ergebnisse der Nutzungsanalyse anzusehen. Die Nutzung elektronischer Fachinformation liegt den Ergebnissen zufolge in einem Verhältnis von $80 / 25$. Bibliotheken bieten ein inhaltlich größeres Spektrum an, welches jedoch in einem derzeit für die untersuchten Fachgebiete nur als unbefriedigend zu bezeichnendem Maße genutzt wird.

Es ist anzunehmen, dass durch die derzeitigen Bezugformen die Konzentrationsprozesse im Verlagswesen befördert werden. Dies deshalb, weil Nutzer auf das in der Bibliothek vorhandene große Titelspektrum, welches durch den additional access und den cross access erworben wird, vorrangig zugreifen und diese Zeitschriften für ihre Arbeiten verwerten. Dies wiederum erhöht die Zitationsrate der entsprechenden Zeitschriften und führt zu einem entsprechenden Ranking dieser Zeitschriften in den Citation Indices, was wiederum die Nutzung für die Forschung erhöht und die Position der Verlage stärkt. Darüber hinaus muss kritisch angemerkt werden, dass es sich bei dieser Zugriffsstruktur nicht länger um einen bedarfsorientierten Bestandsaufbau handelt, sondern Bibliotheken der Gefahr ausgesetzt sind, sich zu umfassenden Distributionskanälen der kommerziellen Verlage zu entwickeln. Sie machen sich zu Erfüllungsgehilfen einer Verlagspolitik, die sich in der Zukunft nachteilig für sie auswirkt.

Der Handlungsspielraum der Konsortien bei der Lizenzierung elektronischer Zeitschriften ist äußerst gering. Die Konsortien selbst gehen nicht davon aus, dass die Verlage in Zukunft neue Geschäftsmodelle entwickeln werden, die zu einer Entlastung der finanziellen Situation in den Bibliotheken beitragen. Derzeit befinden sich Bibliotheken in einer Übergangsphase von der Printumgebung zum digitalen Medium. Nach Einschätzung eines Experten bieten sich den Verlagen in einer ausschließlich digitalen Umgebung neue Möglichkeiten der Preisdifferenzierung, die sich stärker auf die einzelne Bibliothek und das Nutzungsverhalten beziehen. So geht McCabe [29] davon aus, dass die Verlage ein starkes Interesse an Einzelverträgen mit Universitäten haben, da dies den Vorteil bietet, den Preis für das einzelne Produkt nach der Nutzung und dem Budget der Bibliothek variieren zu können. War der Preis für das einzelne Produkt in der Printwelt noch einheitlich, so lässt sich dieser in einer ausschließlich elektronischen Umgebung in Abhängigkeit
Angebots-, Nutzungs- und Bezugsstrukturen elektronischer

Fachinformation in Deutschland 
(-) Angebots-, Nutzungs- und Bezugsstrukturen elektronischer Fachinformation in Deutschland vom angenommenen Budget und vom Stellenwert der Universität variieren. Die Bündelung von Zeitschriften in sogenannte Titelpakete ist auch in einer digitalen Umgebung für die Verlage von Interesse. Einzelverträge mit den Universitäten in Verbindung mit Titelpaketen sowie die geringen Folgekosten in der digitalen Umgebung tragen zu einer Erhöhung der Gewinne kommerzieller Verlage bei. Die unverändert hohen und noch steigenden Preise für elektronische Zeitschriften [30] zeigen, dass konsortiale Zusammenschlüsse nicht das geeignete Instrument sind, um entscheidenden Einfluss auf die Preispolitik der Verlage zu nehmen, die in einigen Wissenschaftsfeldern als Oligopolisten agieren. Hieran ändern auch die beschriebenen Strategien der Kostenreduzierung wenig. Vor diesem Hintergrund ist es nicht erstaunlich, dass für die Konsortien die Finanzierung der Lizenzen für elektronische Ressourcen und die Entwicklung von Lizenzmodellen derzeit die Hauptprobleme darstellen [31].

\section{Ausblick}

Die vorliegenden Ergebnisse machen deutlich, dass es zwischen den Anbietern und den Abnehmern elektronischer Fachinformation Interessenund Zielkonflikte gibt. Dies liegt an den verschiedenen Kulturen der beteiligten Akteure. Das Interesse der WissenschaftlerInnen zielt auf eine Verbreitung der wissenschaftlichen Erkenntnisse und die damit verbundene Aufmerksamkeit, die für die wissenschaftliche Karriere die Voraussetzung bildet. Das Interesse der großen kommerziellen Verlage zielt auf eine Gewinnmaximierung der Ware Information. Hierdurch wird das traditionell partnerschaftliche Verhältnis zwischen den beteiligten Akteuren nachhaltig gestört. Die gegenwärtigen Bezugsformen tragen langfristig nicht zu einer Kostensenkung für elektronische Fachinformation bei.

Die Empfehlungen der Hochschulrektorenkonferenz aus dem Jahre 2002 verdeutlichen jedoch, dass die Problematik der wissenschaftlichen Informationsversorgung die Ebene der Hochschulrektoren erreicht hat. Neben dem Aufund Ausbau hochschuleigener Publikationsserver wird die Entwicklung und Umsetzung neuer "digitaler" Wertschöpfungsketten thematisiert, die dazu beitragen können, das gestörte Gleichgewicht zwischen den Akteuren wiederherzustellen bzw. die wissenschaftliche Kommunikation auf eine von Verlagen unabhängigere Basis zu stellen. Die Durchsetzung neuer $\mathrm{Ge}$ schäftsmodelle ist in hohem Maße von der Entwicklung und dem Einsatz alternativer Publikationssysteme abhängig. Hierfür bilden die bestehenden Informations- und Kommunikationstechnologien die Voraussetzungen. In diesem Zusammenhang spielen Qualitätssicherung, das Marketing/der Vertrieb dieser Produkte sowie das Lobbying für diese Projekte bei den entsprechenden Akteuren eine zentrale Rolle:

Qualitätssicherung: In der traditionellen Wertschöpfungskette werden die Begutachtungen durch universitätsübergreifende und internationale Gremien, den Editorial Boards vorgenommen. Die hierfür koordinierende Tätigkeit wird durch die Verlage sichergestellt. Der zukünftige Erfolg alternativer Publikationssysteme ist maßgeblich von der Bereitschaft der Wissenschaftler abhängig, diese aktiv zu nutzen und auch als Gutachter in neuen elektronischen Zeitschriften tätig zu werden. Neben der Entwicklung einer technologischen Infrastruktur müssen auch offene und beschleunigte Peer-Review-Verfahren zum Einsatz kommen. Marketing/Vertrieb: Für den Erfolg zukünftiger Verlagsprodukte aus Hochschulen spielt das Marketing und der Vertrieb eine zentrale Rolle.
Festzustellen ist, dass die Bedeutung in derzeitigen Ansätzen verlegerischer Tätigkeit durch Hochschulen vernachlässigt wird.

Lobbying: Solange die Weiterverwertungsrechte keine Änderung erfahren haben, ist es notwendig, für alternative Publikationssysteme an Hochschulen massive Lobbyarbeit zu betreiben. Dies setzt die Einbeziehung der Universitätsleitung und Professoren der Universität voraus, die sich verstärkt für die Nutzung bestehender Publikationssysteme und die Entwicklung neuer wissenschaftlicher Kommunikationsstrukturen einsetzen müssen.

\section{Literaturverzeichnis}

[1] vgl. hierzu ausführlicher Meier, Michael (2002): Returning Science to the Scientists. Der Umbruch im STM-Zeitschriftenmarkt unter Einfluss des Electronic Publishing, München S.25ff

\section{[2] Ebd.}

[3] vgl. zum Zusammenhang von Verlagsfusionen und Preissteigerungen. McCabe, Mark (2000): Academic Journal Pricing and Market Power. A Portfolio Approach. http://www.prism.gatech edu/ mm284/JournPub.PDF (Zugriff: 1.5.2002)

[4] vgl. hierzu ausführlicher: Roosendaal, Hans; Geurts, Peter; Vet van der, Paul (2002): Eine neue Wertschöpfungskette für den Markt wissenschaftlicher Information. In: Bibliothek. Forschung und Praxis, .26 Jg., Nr.2, S.149-153

[5] In der Stichprobe befinden sich 25 Universitätsbibliotheken, 21 Fachhochschulbibliotheken, 6 Landes- bzw. Regionalbibliotheken und $2 \mathrm{Na}$ tionalbibliotheken. Das Zeitschriftenangebot umfasst 3.679 Zeitschriftentitel und 53.147 Zeitschriftenexemplare in elektronischer Form und in Printausgaben. 
[6] Bei einer Zuordnung der Datenbank Medline zu dem Fachgebiet Psychologie verteilt sich die Anzahl der $\mathrm{Li}$ zenzen wie folgt auf die Bibliothekstypen: Universitätsbibliothek: 67 Lizenzen; Nationalbibliothek: 0 Lizenzen, Regionalbibliothek: 5 Lizenzen, Fachhochschulbibliothek: 8 Lizenzen

[7] Berücksichtigt werden nur kostenpflichtige Datenbankprodukte, die in mehr als 10 wissenschaftlichen Bibliotheken vorhanden sind

[8] vgl. hierzu Dugall, Berndt, Fladung, Rainer (2002): Empirische Analyse der Nutzung elektronischer Zeitschriften. URL: http://www.m-lehrstuhl de/personal/personaldetails php?pernr $=24$

[9] Als Nutzungsfall wird der Volltextzugriff in der jeweiligen Form (HTML oder PDF) angesehen. Zugriffe auf Inhaltsverzeichnisse oder $\mathrm{Zu}$ sammenfassungen werden nicht berücksichtigt

[10] Hierbei handelt es sich um ein statistisches Verteilungsmaß, welches zwischen 0 und 1 normiert ist. Je gröBer der Wert ausfällt, desto ungleicher ist die Verteilung

[11] Schreiben der Hochschulrektorenkonferenz (2001): Reduzierung der Etatkrise wissenschaftlicher Bibliotheken durch Konsortialverträge, S. 1

[12] Ebd.

[13] Ebd.

[14] German, Austrian and Swiss Consortia Organisation. URL: http://www.hbz-nrw.de/ kunden/gast/konsortien/mitglieder.html

\section{[15] Stand: 1.7 .2002}

[16] Die Ebene des lokalen Erwerbs bleibt bei den folgenden Ausführungen unberücksichtigt, da gemeinschaftliche Einkaufsformen im Vordergrund stehen.
[17] vgl. Reinhardt Werner (2002): Arbeitsgemeinschaft Deutscher, Österreichischer und Schweizer Konsortien (German, Austrian and Swiss Consortia Organisation: GA$\mathrm{SCO}$ ):Bericht über die Aktivitäten 2001-2002. In: Bibliotheksdienst $36 . \mathrm{Jg}$. H.10, S. 1374-1377

[18] Piguet, Arlette (2002): Das Konsortium der Schweizer Hochschulbibliotheken auf Erfolgskurs. In: Digitale Medizinbibliotheken. In: Medizin, Bibliothek, Information. Vol.2, Nr.2

http://www.akh-wien.ac.at/ $\mathrm{agmb} / \mathrm{mbi} / 2002 \_2 / 44-45 \mathrm{pi}-$ guet.pdf (Zugriff: 1.6.2002)

[19] vgl. Schweizerische Universitätskonferenz. Generalsekretariat Reglement des Konsortiums der Schweizer Hochschulbibliotheken. 8/01A

[20] zur Beschlussfähigkeit vgl. ausführlicher Schweizerische Universitätskonferenz. Generalsekretariat: Reglement des Konsortium der Schweizer Hochschulbibliotheken. 8/01A vom 22.2.2001, S.4

[21] http://www.ukb.nl

[22] Befragt wurde die Arbeitsgruppe Konsortialfragen Thüringen, die Arbeitsgruppe Konsortien Sachsen, das baden-württembergische Konsortium, das bayerische Konsortium, das FriedrichAlthoff-Konsortium, das $\mathrm{He}$ BIS-Konsortium, das Konsortium der Niederlande, das Konsortium Nordrhein-Westfalen und das Konsortium der Schweiz. Erhebungszeitraum ist das Jahr 2001

[23] Relativer Anteil am Gesamtumsatz in \%

[24] Nordrhein-Westfalen, Baden-Württemberg, Bayern, UKB und die Max-PlanckGesellschaft machen keine Angaben

[25] Relativer Anteil am Gesamtvolumen der Verträge in \%

[26] Anzahl der Nennungen:
16, Mehrfachnennungen möglich

[27] vgl. hierzu ausführlicher Fladung, Rainer; Dugall, Berndt (2002): Entscheidungsorientierte Kostenbetrachtung für den Bezug elektronischer Zeitschriften im konsortialen Rahmen anhand ausgewählter Beispiele, in: ABI Technik, Heft 1 , S.316-340

[28] Grundsätzlich muss bei dieser Kostenrechnung angemerkt werden, dass sowohl der Kostensatz/Zugriff auf einen Volltext als auch die Wachstumsrate hinsichtlich der Nutzung geschätzt sind

[29] Expertengespräch mit Mark McCabe geführt am 16.12.2002

[30] vgl. hierzu die Pressemitteilung des Deutschen Bibliotheksverbandes vom 24. 09 2002

[31] 13 von 21 Nennungen DFG-Projekt: Perspektiven zu Angebots- und Bezugsformen für elektronische Informationsressourcen in der Bundesrepublik Deutschland

Anschrift der Verfasser:

Dr. Andreas Degkwitz

Amt. Direktor der Universitätsbibliothek Potsdam

Am Neuen Palais 10

Haus 11

14469 Potsdam

degkwitz@rz.uni-potsdam.de

Tel.: (03 31) 9 77-12 49

Fax: (03 31) 9 77-14 58

Dipl.Soz. Heike Andermann

Bibliotheksassessorin

Universitätskomplex $I I I$,

Haus 5

August-Bebel-Strasse 89

14482 Potsdam

anderman@rz.uni-pots-

dam.de

Tel.: (0331) $977-3304$

Fax: (03 31) $977-3816$
Angebots-, Nutzungs- und Bezugsstrukturen elektronischer Fachinformation in Deutschland 


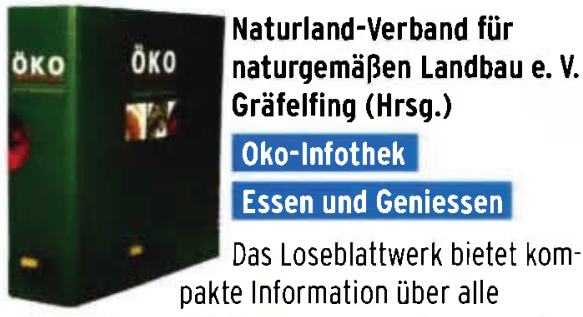

Lebensmittelgruppen, sowie Anbau und Verarbeitung von Lebensmitteln nach den Richtlinien des anerkannt ökologischen Landbaus.

Loseblattwerk, Grundwerk, 265 Blätter $40,00 €$

\section{Getschmann, Dirk/ \\ Scheer, Heinz-Detlef \\ Kleines abc \\ der betrieblichen \\ WeiterbilduI}

Ein "respektloser" Wegweiser für Laien und Profis des betrieblichen Bildungswesens. Ein Helfer durch den Begriffdschungel und Türöffner zum Dialog mit Profis.

•............................ 137 Seiten, illustriert

$5,00 €$

\section{Die Macht der Mitarbeiter}

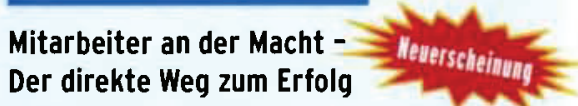

Mitarbeiter an der Macht bedeutet, denjenigen die Macht zu geben, die am schnellsten Änderungen, Firmenphilosophien, Ziele und Strategien umsetzen können. Es heißt loslassen und vertrauen. Es heißt mitárbeiten statt

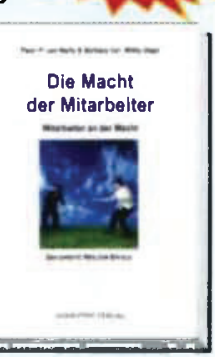
dagegen arbeiten. Wie das funktionieren kann, finden Sie in diesem bemerkenswerten Buch. Dieses Buch hilft Ihnen, Ihren persönlichen und beruflichen Erfolg zu sichern. Die Freude an Dienstleistungen neu zu entfachen, zu steigern und eine langfristige, spannende Bindung einzugehen. Es soll Wege aufzeigen, mit dem steigenden Konkurrenzkampf umzugehen. Es soll richtungsweisend für all jene sein, die in der Dienstleistungsbranche arbeiten. •.......................... 174 Seiten

Scheer, Heinz-Detlef Enfolnreichestratenion. fij starke Teams Gegenseitige Teamberatung im Betrieb

Ziel des Buches ist es, den Leser in die Lage zu versetzen, zusammen mit interes. sierten Kollegen einen Kreis von „Problemlösern" zu gründen, der Probleme angeht, die im eigenen Umfeld entstehen.

149 Seiten, Paperback
Dr. med. Fabritius, Peter/

Dr. med. Koch-Wrenger, Klaus Dieter/Dr. med. Otto, Olaf Gesundheitsschut?

in Beruf und auf Reisen Dieses Buch stellt im allgemeinen Teil ausgewählte, für die betriebliche Praxis wichtige gesetzliche Mindestvorschriften der Arbeitssicherheit und der Arbeitsmedizin vor. Darüber hinaus wird das Thema „Reisemedizin" umfassend behandelt.

227 Seiten

$25,00 €$

\section{Bierschenck/Lenfers/ Lüttke/Dr. Siepe/Dr. Tiele \\ Der Seminarberater}

ob kleines Seminar oder großer Kongress, ob Außendiensttagung oder Produktvorstellung im kleinen Kreis. Der Seminarberater ist deshalb das unentbehrliche Hilfsmittel für jeden, der eine erfolgreiche Veranstaltung planen und durchführen möchte.

76 Seiten, Paperback

$10,00 €$

Yvonne Lüscher Baglioni

Seminar-Unterlagen

\section{Optimal Gestalten}

Diese aktuelle Loseblatt-

sammlung leistet Hilfe-

stellung bei der richtigen

Konzeption von Seminar-

und Tagungsunterlagen.

Ein Top-Ratgeber für alle,

die Seminare planen und

durchführen.

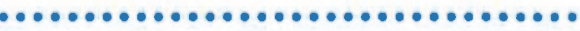

Grundwerk mit 200 Seiten

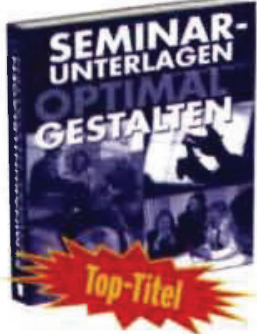

$50,00 €$

"Alten- und Pflegeheims

Das neue Adressbuch enthält die Adressen der deutschen Alten- und Pflegeheime. Insgesamt wurden 7.361 Heime in dieses neue Branchenbuch eingetragen. Besonderen Wert wurde auf die Veröffentlichung von Führungspersonal und den Service-

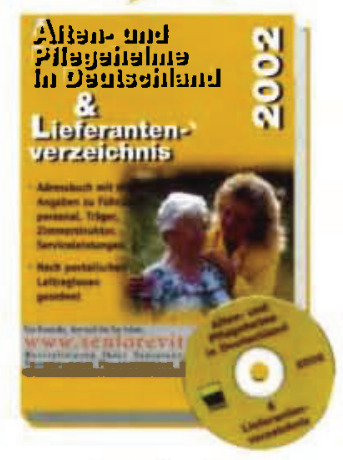

leistungen gelegt, die von sehr vielen Heimen angegeben wurden. Damit kann sich der Leser und Nutzer dieses Branchenbuches einen Überblick über die diversen Leistungen verschaffen, die für ihn oder für seine Angehörigen bei der Suche nach einem Altenheim wichtig sind (u. a. Baujahr, Gesamtplätze, Verkehrsanbindungen, Pflegeeinrichtungen, Pflegeplätze,

Zimmerstruktur, Wohnungen, Appartements, Tierhaltung, u.v.m.)

$10,00 € 256$ Seiten, mit CD-ROM Besgit,00

Dr. Fröhlich, Peter

Kritisieren - aber richtig

Kritik wirkt in der Realität oft überzogen oder verletzend oder sie bleibt gar ganz aus, obwohl sie angebracht wäre. Dieses Buch soll eine Hilfe für all jene sein, die Kritik aussprechen müssen oder

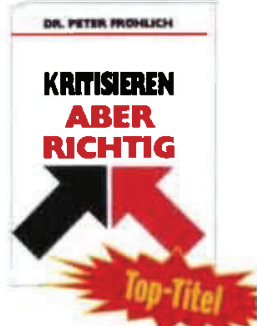
müssten, denn Fehler sind oft Orientierungshifen. Dabei soll der Nutzen von Kritik erkannt und im Gespräch konstruktiv angebracht werden.

151 Seiten, Paperback

$25,00 €$

\section{Völlig aktualisierte Neuauflage! DeGefest Ratgeber 2003/2004 \\ Tagungsstätten im Herzen Europas}

Dieser einzigartige

Ratgeber verhilft

Ihnen zu einer erfol-

greichen Tagung und

unterstützt Sie

bei der Suche nach

einer geeigneten

Tagungsstätte. Alle

Tagungsstätten werden umfassend präsentiert und entsprechen räumlich und nach der techni-

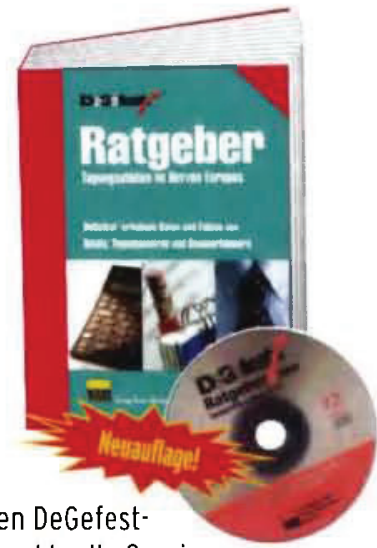
schen Ausstattung den DeGefestQualitätskriterien. Ein aktuelle Serviceteil mit Land- und Straßenkarten, ein Lexikon für die Kongress-, Tagungs- und Messewirt schaft, eine Anleitung zur Planung und Durchführung einer Veranstaltung komplettieren diesen wertvollen Ratgeber.

256 Seiten,

inkl. CD-ROM

$15,00 €$

\section{Jerzy Jaworski, Maik Nossek}

Eny-Pronramme

für die professionelle

Kongressorganisation

\section{in Veroleich}

Ziel dieser Neuerscheinung ist es, auf der Basis eines fundier ten Anforderungs-kataloges eine vergleichende und bewer-

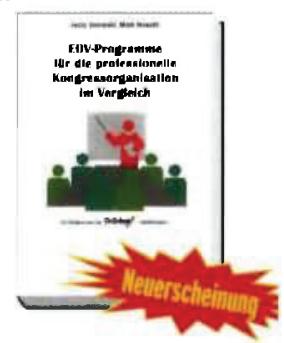

tende Gegenüberstellung der zur Zeit auf dem deutschen Markt angebotenen branchenspezifischen Softwarelösungen anzufertigen, welche für die professionelle Organisation von Kongressen, Tagungen und Seminaren geeignet sind. Das Buch richtet sich an Anwender, welche die Planung, Vorbereitung, Durchführung und Nachbereitung von Kongressen oder sonstigen Veranstaltungen dieser Art als tägliches Geschäft betrachten.

182 Seiten

$40,00 €$

\section{Alle Preise inc. UwSt. und 2201. Versandkosten.}

Bestellanschrift:

Stuttgarter Verlagskontor

SVK-GmbH - Abt. VA VNM

Postfach $106016 \bullet 70049$ Stuttoart

Telefon: $0711 / 6672-1924$ - Telefax: $0711 / 6672-1974$

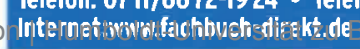

